\title{
Global solutions and asymptotic behavior for a parabolic degenerate coupled system arising from biology
}

\author{
Gabriela Liţcanu* and Cristian Morales-Rodrigo** \\ *Institute of Mathematics "O. Mayer", Romanian Academy, \\ 700505 Iaşi, Romania. e-mail: litcanu@uaic.ro \\ Institute of Mathematics, Witten/Herdecke University, \\ D-58453 Witten, Germany. \\ **Institute of Applied Mathematics and Mechanics, Warsaw University, \\ 02-097 Warsaw, Poland. e-mail: cristianmatematicas@yahoo.com
}

\begin{abstract}
In this paper we will focus on a parabolic degenerate system with respect to unknown functions $u$ and $w$ on a bounded domain of the two-dimensional Euclidean space. This system appears as a mathematical model for some biological processes. Global existence and uniqueness of a nonnegative classical Hölder continuous solution are proved. The last part of the paper is devoted to the study of the asymptotic behavior of the solutions.
\end{abstract}

AMS Subject Classifications: 35B30, 35B40, 35B45, 92C17

Keywords: parabolic-degenerate system, global existence, classical solutions, steady state, asymptotic behavior.

\section{Introduction}

During the last years models originated from biology earned a privileged place in mathematical modeling and became the focus of interest of mathematicians and biologists as well. In many cases the study of these models involves challenging mathematical problems that originate in the intrinsic mathematical structure of the model. Moreover the possibility of taking suitable hypotheses is limited by the necessity to fit with experimental data of the process the model originates in.

Let us consider the following initial-boundary problem:

$$
\begin{array}{ll}
\frac{\partial u}{\partial t}=a \Delta u-b \nabla \cdot(u \chi(w) \nabla w)+f(u, w) & x \in \Omega, \quad t \in \mathbb{R}_{+} \\
\frac{\partial w}{\partial t}=-k w^{\beta} u & x \in \Omega, \quad t \in \mathbb{R}_{+} \\
u(x, 0)=u_{0}(x) \geqslant 0 & x \in \Omega \\
w(x, 0)=w_{0}(x)>0 & x \in \Omega
\end{array}
$$

where $\Omega \subseteq \mathbb{R}^{N}$ is a domain, $a, b$ and $k$ are positive constants, $\chi(w)=w^{-\alpha}, 0 \leqslant \alpha<1, \beta \geqslant 1$ and $f$ is a given function. If $\Omega$ is bounded, then the system (1.1)-(1.4) is considered together with the no-flux boundary condition

$$
\frac{\partial u}{\partial \eta}-u \chi(w) \frac{\partial w}{\partial \eta}=0 \quad x \in \partial \Omega, \quad t \in \mathbb{R}_{+}
$$


where $\eta$ denotes the unit outward normal vector of $\partial \Omega$.

This system is a particular version of the well-known mathematical model proposed by Keller and Segel [19] (see also [17, 18, 20]) with an additional reaction term $f(u, w)$ in the first equation. The KellerSegel model was proposed in order to describe the spatial aggregation of cellular slime molds which move toward high concentrations of some chemical substance secreted by the cells themselves. The function $u(x, t)$ describes the density distribution of the cell population, $w(x, t)$ denotes the concentration of the chemical substance at a position $x \in \Omega$ and a time $t \in \mathbb{R}_{+}$and the function $\chi$ is the chemotactic sensitivity. The classical Keller-Segel model, when the second variable is also supposed to be diffusive, has been subject of many papers (see, for example, the surveys of Horstmann [15, [16] and the references given therein). In the literature there are many theoretical results for the Keller-Segel model concerning existence and uniqueness as well as the qualitative behavior of the solutions. Most of the results were focused on the global existence of solutions versus blow-up in finite time. Both behaviors strongly depend on the initial data and space dimension.

The system (1.1)-(1.5) also appears as a simplified mathematical model describing the tumor growth when the formation of new blood vessels from the pre-existing vascular network is initiated (angiogenesis). In this case, the function $u(x, t)$ describes the tumor cells density and $w(x, t)$ denotes the density of the extracellular matrix (the surrounding healthy tissue degraded locally by the action of tumor cells). There are several models of different stages of the angiogenesis process incorporating also the action of some degradative enzymes, cell cycle elements or cell age structures. For a more thorough biological background and numerical results concerning the angiogenesis process see, for example, 2], 3], 4], 22, 23. We refer also to [28, 29] where the global existence and uniqueness of solutions in the case of some systems related with this process are investigated.

Previously, a version of the system (1.1)-(1.5) was studied by Rascle in [25] (see also [24]) with the boundary condition (1.5) replaced with

$$
\frac{\partial u}{\partial \eta}=0 .
$$

Instead of (1.4) he takes a positive constant as initial condition for the function $w, w_{0}(x) \equiv w_{0}>0$ and $f(u, v)$ satisfying the following condition

$$
\exists L>0, \forall u \in \mathbb{R}, \forall w>0,|f(u, w)| \leqslant L|u| .
$$

In the previous hypotheses, the local existence and uniqueness of a classical Hölder continuous solution of the system (1.1)-(1.4) has been proved when $\Omega \subset \mathbb{R}^{N}$ is a bounded domain with smooth boundary $\partial \Omega$. The global existence has been shown in one space dimension. We mention that another result, in the one dimensional space, concerning the global existence and uniqueness of classical solutions for a similar system is given in 12 .

In more than one dimension, when $\Omega$ is the whole space $\mathbb{R}^{N}$, the system (1.1)-(1.5) has been considered in [5], 6], 7] with $\chi(w)$ a given positive function on $\mathbb{R}_{+}$such that $w \chi(w)$ is strictly increasing (thus including the case $\left.\chi(w)=w^{-\alpha}, 0 \leqslant \alpha<1\right)$ and $f \equiv 0$. In this case the global existence of weak solutions has been proved.

In 13 the authors considered the problem (1.1)-(1.4) in a more general form under Dirichlet conditions. Assuming that a priori $L^{\infty}$ bounds are available they proved the local and global existence of weak solutions.

Finally, we cite here the paper [27] where the author considers instead of the equation (1.2) the following one

$$
\frac{\partial w}{\partial t}=g(u, w)
$$

but under some hypotheses on $g$ that are not satisfied in the case we shall consider in this paper (see also [9], [10]).

Our aim in this paper is to prove the global existence in time and uniqueness of a classical Hölder continuous solution for the problem (1.1)-(1.4) when $\Omega \subset \mathbb{R}^{2}$ is a bounded domain with smooth boundary $\partial \Omega$ and the reaction term is the logistic growing function. Also the long time asymptotic behavior of the 
solution is investigated. In order to simplify the presentation of the results, we shall consider in what follows the case $\alpha=0$. The more general cases $\beta \geqslant 1,0 \leqslant \alpha<1$ (or even when the function $\chi$ is a more general decreasing function) can be treated similarly, the estimations being more tedious.

This paper is organized as follows. In Section 2 we review some basic facts concerning the notations and terminology used through the paper and we also give some auxiliary results. The proof of the local existence in time and uniqueness of a classical solution is accomplished by applying a fixed point argument in a suitably chosen function space and is presented in Section 3.

In Section 4 we will be concerned with the global existence in time of the classical solutions and for this we will begin by establishing a priori bounds.

In Subsection 4.1 we obtain a Lyapunov function for the system (independent of the space dimension) by an analogous method as in [6] (see also [7, 11, 14]). We derive $L^{p}(\Omega)$ estimates independent on time in Subsection 4.2. After establishing a priori $L^{\infty}(\Omega)$ uniform bounds in Subsection 4.3, we proceed to prove the existence of global Hölder continuous solutions imposing that the initial data are smooth enough.

Section 5 is devoted to the study of the long time asymptotic behavior of the solutions. More precisely, we prove that the solution converges to a steady state of the system, exponentially if $\beta=1$ and at a polynomial rate if $\beta>1$.

\section{Preliminaries}

Hereafter we assume that $\Omega \subset \mathbb{R}^{N}, N \geqslant 1$ is a bounded domain with smooth boundary $\partial \Omega \in C^{l+2}\left(\mathbb{R}^{N-1}\right)$. Given $T \in(0,+\infty]$, we consider the cylindrical domain denoted by $\Omega_{T}=\Omega \times(0, T)$ with lateral surface $\partial \Omega_{T}=\partial \Omega \times(0, T)$.

We are using in this paper the standard notation of function spaces. $L^{p}(\Omega)$ and $W^{m, p}(\Omega)$ with $1 \leqslant p \leqslant \infty$, $m \geqslant 1$ are the Lebesgue spaces and respectively, Sobolev spaces of functions on $\Omega$. For a general Banach space $X$, its norm is denoted by $\|\cdot\|_{X}$. The space $L^{p}(0, T ; X)$ is the Banach space of all Bochner measurable functions $f:(0, T) \rightarrow X$ such that $\|f\|_{X} \in L^{p}(0, T)$.

For a positive integer $n$ we consider the Banach space $W_{p}^{2 n, n}\left(\Omega_{T}\right)=\left\{f ; D_{t}^{r} D_{x}^{s} f \in L^{p}\left(\Omega_{T}\right), 2 r+|s| \leqslant 2 n\right\}$ together with the norm

$$
\|f\|_{p, \Omega_{T}}^{(2 n)}=\sum_{0 \leqslant 2 r+|s| \leqslant 2 n}\left\|D_{t}^{r} D_{x}^{s} f\right\|_{L^{p}\left(\Omega_{T}\right)} .
$$

Given a non-integer positive number $0<l<1$, we denote by $C^{l+i, l / 2+i / 2}\left(\bar{\Omega}_{T}\right), i=1,2$ the Hölder space of exponents $l+i$ and $l / 2+i / 2$ by respect to $x$, respectively $t$ of continuous and bounded functions $\{f(x, t)\}$ defined on $\bar{\Omega}_{T}$, provided with continuous and bounded derivatives $\left\{D_{t}^{r} D_{x}^{s} f(x, t)\right\}$ for $2 r+|s| \leqslant i$. It is endowed with the norm given by

$$
\begin{aligned}
|f|_{\Omega_{T}}^{(l+i)} & :=\sum_{2 r+|s|=i}\left\langle D_{t}^{r} D_{x}^{s} f\right\rangle_{x, \Omega_{T}}^{(l)}+\sum_{\max \{0, i-1\} \leqslant 2 r+|s| \leqslant i}\left\langle D_{t}^{r} D_{x}^{s} f\right\rangle_{t, \Omega_{T}}^{((l-2 r-|s|) / 2+i / 2)}+ \\
& +\sum_{0 \leqslant 2 r+|s| \leqslant i} \max _{(x, t) \in \bar{\Omega}_{T}}\left|D_{t}^{r} D_{x}^{s} f\right|
\end{aligned}
$$

where

$$
\langle f\rangle_{x, \Omega_{T}}^{(l)}:=\sup _{\substack{(x, t),\left(x^{\prime}, t\right) \in \bar{\Omega}_{T} \\\left|x-x^{\prime}\right| \leqslant \rho_{0}}} \frac{\left|f(x, t)-f\left(x^{\prime}, t\right)\right|}{\left|x-x^{\prime}\right|^{l}}, \quad\langle f\rangle_{t, \Omega_{T}}^{(l / 2)}:=\sup _{\substack{(x, t),\left(x, t^{\prime}\right) \in \bar{\Omega}_{T} \\\left|t-t^{\prime}\right| \leqslant \rho_{0}}} \frac{\left|f(x, t)-f\left(x, t^{\prime}\right)\right|}{\left|t-t^{\prime}\right|^{l / 2}} .
$$

This norm mentioned above depends on $\rho_{0}>0$, but changing this constant leads to an equivalent norm. Throughout this paper we denote by $C, C_{i}(i=1,2, \ldots)$ positive constants which are independent of time, but we shall indicate explicitly on which other parameters they are dependent, if it will be the case. The constants $C$ are not necessarily the same at different occurrences. 
Some properties for the norms in the Hölder spaces which will be used often in the next sections are given below. Since the proofs are standard, but tedious, we omit the details.

Lemma 2.1 If $f(x, t) \in C^{l+2, l / 2+1}\left(\bar{\Omega}_{T}\right), 0<l<1$, then we have:

(i) $\frac{\partial f}{\partial t} \in C^{l, l / 2}\left(\bar{\Omega}_{T}\right)$,

(ii) $\frac{\partial f}{\partial x_{j}} \in C^{l+1, l / 2+1 / 2}\left(\bar{\Omega}_{T}\right), j=1, \ldots, N$,

(iii) $\Delta f \in C^{l, l / 2}\left(\bar{\Omega}_{T}\right)$,

(iv) $\frac{\partial f}{\partial \eta} \in C^{l+1, l / 2+1 / 2}\left(\bar{\Omega}_{T}\right)$, where $\eta$ denotes the unit outward normal vector of $\partial \Omega$.

Lemma 2.2 If $f(x, t) \in C^{l+2, l / 2+1}\left(\bar{\Omega}_{T}\right), 0<l<1$, then $H(x, t)=\int_{0}^{t} f(x, s) d s \in C^{l+2, l / 2+1}\left(\bar{\Omega}_{T}\right)$. Moreover,

$$
|H|_{\Omega_{T}}^{(l+2)} \leqslant C \max \left\{T, T^{(1-l) / 2}\right\}|f(x, t)|_{\Omega_{T}}^{(l+2)}+|f(x, 0)|_{\Omega_{T}}^{(l)} .
$$

Lemma 2.3 If $f, g \in C^{l+i, l / 2+i / 2}\left(\bar{\Omega}_{T}\right), 0<l<1$, then $f g \in C^{l+i, l / 2+i / 2}\left(\bar{\Omega}_{T}\right)$ and

$$
|f g|_{\Omega_{T}}^{(l+i)} \leqslant C|f|_{\Omega_{T}}^{(l+i)}|g|_{\Omega_{T}}^{(l+i)}
$$

for $i=0,1,2$.

Lemma 2.4 ([25], Lemma 1) Let $\varphi, \psi: \Omega_{T} \rightarrow K \subset \mathbb{R}^{N}$, where $K$ is a compact in $\mathbb{R}^{N}$, be two functions in $\left(C^{l+2, l / 2+1}\left(\bar{\Omega}_{T}\right)\right)^{N}$ and let $f \in C^{3}(K)$. Then $f \circ \varphi$ and $f \circ \psi$ are in $C^{l+2, l / 2+1}\left(\bar{\Omega}_{T}\right)$ and we have

$$
|f \circ \varphi-f \circ \psi|_{\Omega_{T}}^{(l+2)} \leqslant \Phi\|f\|_{C^{3}(K)}\left(|\varphi-\psi|_{\Omega_{T}}^{(l+2)}\right)^{\gamma}
$$

where $\gamma=\min \{l / 2,1-l\}$ and $\Phi=\Phi\left(|\varphi|_{\Omega_{T}}^{(l+2)},|\psi|_{\Omega_{T}}^{(l+2)}\right)$ is an increasing function on both its arguments.

The remaining of this section is devoted to some general results for the existence of solutions for parabolic equations. We consider the problem:

$$
\begin{array}{ll}
\frac{\partial u}{\partial t}-\Delta u+\sum_{i=1}^{N} a_{i}(x, t) \frac{\partial u}{\partial x_{i}}+a(x, t) u=F(x, t) & (x, t) \in \Omega_{T} \\
\frac{\partial u}{\partial \eta}-u \frac{\partial g}{\partial \eta}(x, t)=G(x, t) & (x, t) \in \partial \Omega_{T} \\
u(x, 0)=u_{0}(x) & x \in \Omega .
\end{array}
$$

Let us remark that, if we make the change of variables $v(x, t)=u(x, t) e^{-g(x, t)}$ the system (2.4)-(2.6) becomes:

$$
\begin{array}{ll}
\frac{\partial v}{\partial t}-\Delta v+\sum_{i=1}^{N} b_{i}(x, t) \frac{\partial v}{\partial x_{i}}+b(x, t) v=\widetilde{F}(x, t) & (x, t) \in \Omega_{T} \\
\frac{\partial v}{\partial \eta}=\widetilde{G}(x, t) & (x, t) \in \partial \Omega_{T} \\
v(x, 0)=v_{0}(x) & x \in \Omega
\end{array}
$$


where the coefficients are given by:

$$
\begin{aligned}
& b_{i}(x, t)=a_{i}(x, t)-2 \frac{\partial g}{\partial x_{i}}(x, t), \quad 1 \leqslant i \leqslant N \\
& b(x, t)=a(x, t)+\frac{\partial g}{\partial t}(x, t)-\Delta g+\sum_{i=1}^{N} a_{i}(x, t) \frac{\partial g}{\partial x_{i}}(x, t)-\sum_{i=1}^{N}\left(\frac{\partial g}{\partial x_{i}}(x, t)\right)^{2} \\
& \widetilde{F}(x, t)=F(x, t) e^{-g(x, t)} \\
& \widetilde{G}(x, t)=G(x, t) e^{-g(x, t)} \\
& v_{0}(x)=u_{0}(x) e^{-g(x, 0)} .
\end{aligned}
$$

Theorem 2.5 (24], Theorem II.2) Let $0<l<1$ and $\Omega \subset \mathbb{R}^{N}$ be a bounded domain with the boundary $\partial \Omega \in C^{l+2}$ and $0<T<\infty$. We suppose that the following hypotheses are satisfied:

- the coefficients $b_{i}(x, t)(1 \leqslant i \leqslant N), b(x, t)$ belong to the space $C^{l, l / 2}\left(\bar{\Omega}_{T}\right)$;

- $\widetilde{F}(x, t) \in C^{l, l / 2}\left(\bar{\Omega}_{T}\right), \widetilde{G}(x, t) \in C^{l+1, l / 2+1 / 2}\left(\overline{\partial \Omega}_{T}\right)$ and $v_{0}(x) \in C^{l+2}(\bar{\Omega})$;

- the compatibility condition $\frac{\partial v}{\partial \eta}(x, 0)=\widetilde{G}(x, 0)$ is satisfied for every $x \in \partial \Omega$.

Then the problem (2.7)-(2.9) has a unique solution $v(x, t) \in C^{l+2, l / 2+1}\left(\bar{\Omega}_{T}\right)$ which verifies

$$
|v|_{\Omega_{T}}^{(l+2)} \leqslant \Theta\left(|\widetilde{F}|_{\Omega_{T}}^{(l)}+|\widetilde{G}|_{\partial \Omega_{T}}^{(l+1)}+\left|v_{0}\right|_{\Omega}^{(l+2)}\right)
$$

where $\Theta=\Theta(T, \mu(T))$ is an increasing function on $T$ and on the quantity

$$
\mu(T)=\sum_{i=1}^{N}\left|b_{i}(x, t)\right|_{\Omega_{T}}^{(l)}+|b(x, t)|_{\Omega_{T}}^{(l)} .
$$

Theorem 2.6 Let $0<l<1$ and $\Omega \subset \mathbb{R}^{N}$ be a bounded domain with the boundary $\partial \Omega \in C^{l+2}$ and $0<T<\infty$. We suppose that the following hypotheses are satisfied:

- the coefficients $a_{i}(x, t)(1 \leqslant i \leqslant N), a(x, t)$ belong to the space $C^{l, l / 2}\left(\bar{\Omega}_{T}\right)$;

- $F(x, t) \in C^{l, l / 2}\left(\bar{\Omega}_{T}\right), G(x, t) \in C^{l+1, l / 2+1 / 2}\left(\overline{\partial \Omega}_{T}\right), g(x, t) \in C^{l+2, l / 2+1}\left(\overline{\partial \Omega}_{T}\right)$ and $u_{0}(x) \in C^{l+2}(\bar{\Omega})$;

- the compatibility condition $\frac{\partial u_{0}}{\partial \eta}-u_{0} \frac{\partial g}{\partial \eta}(x, 0)=G(x, 0)$ is satisfied for every $x \in \partial \Omega$.

Then the problem (2.4)-(2.6) has a unique solution $u(x, t) \in C^{l+2, l / 2+1}\left(\bar{\Omega}_{T}\right)$ which verifies

$$
|u|_{\Omega_{T}}^{(l+2)} \leqslant \Psi\left(|F|_{\Omega_{T}}^{(l)}+|G|_{\partial \Omega_{T}}^{(l+1)}+\left|u_{0}\right|_{\Omega}^{(l+2)}\right)
$$

where $\Psi=\Psi\left(T,|g|_{\Omega_{T}}^{(l+2)}, \mu(T)\right)$ is an increasing function in $T$, in $|g|_{\Omega_{T}}^{(l+2)}$ and in the quantity

$$
\mu(T)=\sum_{i=1}^{N}\left|b_{i}(x, t)\right|_{\Omega_{T}}^{(l)}+|b(x, t)|_{\Omega_{T}}^{(l)}
$$

where $b_{i}(x, t)(1 \leqslant i \leqslant N), b(x, t)$ are given by (2.10), (2.11).

Proof. The existence and the uniqueness of the solution is proved in [21], Chapter IV, Theorem 5.3. The only thing that we want to point out is the increasing dependence of the function $\Psi$ on its arguments. From Lemma 2.3 we obtain

$$
|u(x, t)|_{\Omega_{T}}^{(l+2)}=\left|v(x, t) e^{g(x, t)}\right|_{\Omega_{T}}^{(l+2)} \leqslant C|v(x, t)|_{\Omega_{T}}^{(l+2)}\left|e^{g(x, t)}\right|_{\Omega_{T}}^{(l+2)} .
$$

Now, taking into account (2.15) and Lemma 2.4, we obtain immediately the relation (2.16). 


\section{Local existence in time and uniqueness of classical solutions}

As we have already mentioned in Introduction, in order to simplify the presentation of the results, we consider the system (1.1)-(1.5) when $\alpha=0$. We consider, without loss of generality, the normalized system, which means $a=b=k=1$, with the growing source term, more precisely

$$
\begin{array}{ll}
\frac{\partial u}{\partial t}=\Delta u-\nabla \cdot(u \nabla w)+\delta u(1-u) & x \in \Omega, \quad t \in \mathbb{R}_{+} \\
\frac{\partial w}{\partial t}=-w^{\beta} u & x \in \Omega, \quad t \in \mathbb{R}_{+} \\
\frac{\partial u}{\partial \eta}-u \frac{\partial w}{\partial \eta}=0 & x \in \partial \Omega, \quad t \in \mathbb{R}_{+} \\
u(x, 0)=u_{0}(x) \geqslant 0 & x \in \Omega \\
w(x, 0)=w_{0}(x)>0 & x \in \Omega
\end{array}
$$

where $\delta \geqslant 0$ and $\beta \geqslant 1$.

Remark 3.1 In what follows the computations are made for $\beta>1$. The same results are true also for $\beta=1$, but the estimates will be different. We have considered the growing source term, nevertheless the results are valid also in the case of more general functions satisfying appropriate conditions.

The arguments given in this Section are similar to those of Rascle [24, 25]. Because in our case the boundary condition is different and the function $f$ does not satisfy the condition (1.7), we briefly give the proof for the local existence for the sake of completeness.

Let us remark that, if $\beta>1$, we can rewrite the initial problem (3.1)-(3.5):

$$
\begin{array}{ll}
\frac{\partial u}{\partial t}=\nabla\left(\nabla u-u \cdot \nabla\left[\left(w_{0}^{1-\beta}+U\right)^{\frac{1}{1-\beta}}\right]\right)+\delta u\left(1-\frac{1}{\beta-1} \frac{\partial U}{\partial t}\right) & x \in \Omega, \quad t \in \mathbb{R}_{+} \\
\frac{\partial u}{\partial \eta}=u \frac{\partial}{\partial \eta}\left[\left(w_{0}^{1-\beta}+U\right)^{\frac{1}{1-\beta}}\right] & x \in \partial \Omega, \quad t \in \mathbb{R}_{+} \\
u(x, 0)=u_{0}(x) & x \in \Omega \\
U=(\beta-1) \int_{0}^{t} u(x, s) d s & x \in \Omega, \quad t \in \mathbb{R}_{+} \\
U(x, 0)=0 . & x \in \Omega
\end{array}
$$

We consider now the following linear problem in the variable $u$

$$
\begin{array}{ll}
\frac{\partial u}{\partial t}=\Delta u-\sum_{i=1}^{N} a_{i}(x, t) \frac{\partial u}{\partial x_{i}}-a(x, t) u & x \in \Omega, \quad t \in \mathbb{R}_{+} \\
\frac{\partial u}{\partial \eta}=u \frac{\partial g}{\partial \eta} & x \in \partial \Omega, \quad t \in \mathbb{R}_{+} \\
u(x, 0)=u_{0}(x) \geqslant 0 & x \in \Omega
\end{array}
$$

where the coefficients are given by

$$
g(x, t)=\left(w_{0}^{1-\beta}(x)+\phi(x, t)\right)^{\frac{1}{1-\beta}}, \quad a_{i}(x, t)=\frac{\partial g}{\partial x_{i}}, \quad a(x, t)=\Delta g-\delta\left(1+g^{-\beta} \frac{\partial g}{\partial t}\right) .
$$

Theorem 3.1 Let $0<l<1, \Omega \subset \mathbb{R}^{N}$ be a domain with $C^{l+2}$ boundary $\partial \Omega$ and $0<T<\infty$. We suppose that the following hypotheses are satisfied: 
- $\phi \in C^{l+2, l / 2+1}\left(\bar{\Omega}_{T}\right), w_{0} \in C^{l+2}(\bar{\Omega}), u_{0} \in C^{l+2}(\bar{\Omega})$;

- the compatibility condition $\frac{\partial u_{0}}{\partial \eta}(x)=u_{0}(x) \frac{\partial g}{\partial \eta}(x, 0)$ is satisfied for every $x \in \partial \Omega$.

Then the problem (3.11)-(3.13) has a unique nonnegative solution $u(x, t) \in C^{l+2, l / 2+1}\left(\bar{\Omega}_{T}\right)$ which verifies

$$
|u|_{\Omega_{T}}^{(l+2)} \leqslant \Psi\left|u_{0}\right|_{\Omega}^{(l+2)}
$$

where $\Psi=\Psi\left(T,|g|_{\Omega_{T}}^{(l+2)}, \mu(T)\right)$ is an increasing function in $T$, in $|g|_{\Omega_{T}}^{(l+2)}$ and in the quantity

$$
\mu(T)=\sum_{i=1}^{N}\left|a_{i}\right|_{\Omega_{T}}^{(l)}+\left|\frac{\partial g}{\partial t}-\delta\left(1+g^{-\beta} \frac{\partial g}{\partial t}\right)\right|_{\Omega_{T}}^{(l)}
$$

Proof. Taking into account the properties of the norm in Hölder spaces (see Lemma 2.1, Lemma 2.4), we have

$$
a_{i}(x, t) \in C^{l, l / 2}\left(\bar{\Omega}_{T}\right), \quad a(x, t) \in C^{l, l / 2}\left(\bar{\Omega}_{T}\right), \quad g(x, t) \in C^{l+2, l / 2+1}\left(\bar{\Omega}_{T}\right), \quad i=1, \ldots, N
$$

so by Theorem 2.6 we obtain that the problem (3.11)-(3.13) has a unique solution $u(x, t) \in C^{l+2, l / 2+1}\left(\bar{\Omega}_{T}\right)$. Moreover, taking into account (3.14), this solution verifies (3.15).

The nonnegativity of the solution follows from the maximum principle.

We shall prove now the local existence of the solution for the problem (3.1)-(3.5) using a fixed point argument. We consider the set

$$
X(T, \sigma)=\left\{\phi \in C^{l+2, l / 2+1}\left(\overline{\Omega_{T}}\right) ; \quad|\phi|_{\Omega_{T}}^{(l+2)} \leqslant \sigma, \quad \phi(., 0)=0, \quad \phi \geqslant 0 \text { and } \frac{\partial \phi}{\partial t} \geqslant 0 \text { in } \Omega_{T}\right\}
$$

where $\sigma$ is a positive constant. We define the following operators

$$
S: X \rightarrow C^{l+2, l / 2+1}\left(\bar{\Omega}_{T}\right), \quad S(\phi)=u,
$$

where $u$ is the unique solution of the problem (3.11)-3.13), and

$$
R: C^{l+2, l / 2+1}\left(\overline{\Omega_{T}}\right) \rightarrow C^{l+2, l / 2+1}\left(\bar{\Omega}_{T}\right), \quad R(u)=U
$$

where $U$ is given by the relation (3.9).

Let us observe that, in order to find a solution of the problem (3.6)-(3.10), it is enough to find a fixed point for the application

$$
R \circ S: X \rightarrow C^{l+2, l / 2+1}\left(\bar{\Omega}_{T}\right), \quad(R \circ S)(\phi)=R(u)=U
$$

Theorem 3.2 Let $0<l<1, \Omega \subset \mathbb{R}^{N}$ be a domain with $C^{l+2}$ boundary $\partial \Omega$. We assume that the hypotheses of Theorem 3.1 are satisfied and, moreover, we suppose that $\left|u_{0}\right|_{\Omega}^{(l)}<\sigma /(2(\beta-1))$. Then for every $\zeta>0$ there exists $T_{1}>0$ such that, for all $\tau \in\left(0, T_{1}\right]$ the following properties are true:

(i) the closed convex set $X(\tau, \sigma)$ is invariant by $R \circ S$;

(ii) the operator $R \circ S$ satisfies the following inequality in $X(\tau, \sigma)$ with respect to the norm $|\cdot|_{\Omega_{\tau}}^{(l+2)}$ :

$$
|(R \circ S)(\phi)-(R \circ S)(\psi)|_{\Omega_{\tau}}^{(l+2)} \leqslant \zeta\left(|\phi-\psi|_{\Omega_{\tau}}^{(l+2)}\right)^{\gamma}
$$

where $\gamma=\min \{l / 2,1-l\}$. Therefore, $R \circ S$ has a unique fixed point $\phi$ in $X(\tau, \sigma)$. 
Proof. (i) Because $u(x, t)$ is the unique solution of the problem (3.11)-(3.13) and taking into account Lemma 2.2. Theorem 3.1 and the relation (3.15), for every $0<\tau \leqslant T$, we obtain

$$
\begin{aligned}
|(R \circ S)(\phi)|_{\Omega_{\tau}}^{(l+2)} & \leqslant C(\beta-1) \max \left\{\tau^{(1-l) / 2}, \tau\right\} \Psi\left(\tau,|g(x, t)|_{\Omega_{\tau}}^{(l+2)}, \mu(\tau)\right)\left|u_{0}\right|_{\Omega}^{(l+2)}+ \\
& +(\beta-1)\left|u_{0}\right|_{\Omega_{\tau}}^{(l)} .
\end{aligned}
$$

where $C$ is a constant independent on $\tau$. Now, in order to estimate the function $\Psi\left(\tau,|g|_{\Omega_{\tau}}^{(l+2)}, \mu(\tau)\right)$ which appears in (3.19), we estimate first the norm $|g|_{\Omega_{\tau}}^{(l+2)}$ using Lemma 2.4

$$
|g|_{\Omega_{\tau}}^{(l+2)} \leqslant C_{1}\left(\left|w_{0}^{1-\beta}(x)\right|_{\Omega_{\tau}}^{(l+2)}+\sigma\right)^{\gamma}
$$

where $C_{1}=C_{1}\left(\left|w_{0}(x)\right|_{\Omega_{\tau}}^{(l+2)}, \sigma\right)$ and $\gamma=\min \{l / 2,1-l\}$. Taking into account Lemma 2.1 and (3.20) we obtain

$$
\mu(\tau) \leqslant C_{2}+\frac{\delta}{\beta-1} \sigma+\delta
$$

where $C_{2}=C_{2}\left(\left|w_{0}(x)\right|_{\Omega_{\tau}}^{(l+2)}, \sigma\right)$.

From Theorem 3.1 we know that the function $\Psi$ is increasing on $\tau,|g|_{\Omega_{\tau}}^{(l+2)}$ and $\mu(\tau)$, so we obtain from (3.20) and (3.21) for $0<\tau \leqslant T$

$$
\Psi\left(\tau,|g(x, t)|_{\Omega_{\tau}}^{(l+2)}, \mu(\tau)\right) \leqslant \Psi\left(\tau, C_{1}\left(\left|w_{0}^{1-\beta}(x)\right|_{\Omega_{\tau}}^{(l+2)}+\sigma\right)^{\gamma}, C_{2}+\frac{\delta}{\beta-1} \sigma+\delta\right)=: \Lambda(\sigma) .
$$

Finally, from (3.19), we obtain

$$
|(R \circ S)(\phi)|_{\Omega_{\tau}}^{(l+2)}<C(\beta-1) \max \left\{\tau^{(1-l) / 2}, \tau\right\} \Lambda(\sigma)\left|u_{0}\right|_{\Omega}^{(l+2)}+\frac{1}{2} \sigma .
$$

It follows that for $\tau>0$ sufficiently small $X(\tau, \sigma)$ is invariant by $R \circ S$. Let $T_{2}>0$ be sufficiently small, such that, for all $0<\tau \leqslant T_{2}, X(\tau, \sigma)$ is invariant by $R \circ S$.

(ii) Let $\phi, \bar{\phi} \in X\left(T_{2}, \sigma\right)$ and

$$
U=R(u)=(R \circ S)(\phi), \quad \bar{U}=R(\bar{u})=(R \circ S)(\bar{\phi}) .
$$

It is easy to see that the function $z=u-\bar{u}$ satisfies the problem

$$
\begin{array}{ll}
\frac{\partial z}{\partial t}=\Delta z-\sum_{i=1}^{N} a_{i}(x, t) \frac{\partial z}{\partial x_{i}}-a(x, t) z+\bar{F}(x, t) & x \in \Omega, \quad t \in \mathbb{R}_{+} \\
\frac{\partial z}{\partial \eta}=z \frac{\partial g}{\partial \eta}+\bar{G}(x, t) & x \in \partial \Omega, \quad t \in \mathbb{R}_{+} \\
z(x, 0)=0 & x \in \Omega
\end{array}
$$

where $g(x, t)$ is given by (3.14), $\bar{g}(x, t)=\left(w_{0}^{1-\beta}(x)+\bar{\phi}(x, t)\right)^{\frac{1}{1-\beta}}$ and

$$
\bar{F}(x, t)=\nabla(\bar{u} \cdot \nabla(g-\bar{g}))+\frac{\delta}{\beta-1} \bar{u} \frac{\partial}{\partial t}(\phi-\bar{\phi}), \quad \bar{G}(x, t)=\bar{u} \frac{\partial}{\partial \eta}[g-\bar{g}] .
$$

Let us notice that $\bar{G}(x, 0)=0$, so the function $z(x, t)=(u-\bar{u})(x, t)$ satisfies the compatibility condition $\frac{\partial z}{\partial \eta}(x, 0)-z(x, 0) \frac{\partial g}{\partial \eta}=G(x, 0)$. We obtain, taking into account Theorem 2.6

$$
|(R \circ S)(\phi)-(R \circ S)(\bar{\phi})|_{\Omega_{\tau}}^{(l+2)} \leqslant C_{3}(\beta-1) \max \left\{\tau^{(1-l) / 2}, \tau\right\} \Psi(\sigma)\left|u_{0}\right|_{\Omega_{\tau}}^{(l+2)}\left(|\phi-\bar{\phi}|_{\Omega_{\tau}}^{(l+2)}\right)^{\gamma}
$$


where $\gamma=\min \{l / 2,1-l\}$ and $C_{3}=C_{3}(\sigma)$. By taking $\tau$ sufficiently small the inequality (3.18) follows. We choose now $T_{1}<T_{2}$ such that $(i)$ and (ii) are fulfilled for all $\tau \in\left(0, T_{1}\right]$.

We define now the following two sequences

$$
u_{n}=S\left(U_{n}\right), \quad U_{n+1}=R\left(u_{n}\right)=(R \circ S)\left(U_{n}\right)
$$

where $U_{0}=0$. It follows from the above considerations that $\left(U_{n}\right)_{n \in \mathbb{N}}$ is a Cauchy sequence, so it converges to an element $U$, which is a fixed point of $R \circ S$. The inequality (3.18) implies the uniqueness of this fixed point.

The continuity of the application $S$ implies that the sequence $\left(u_{n}\right)_{n \in \mathbb{N}}$ converges to $u=S(U)$. It is easy to see that $(u, U)$ is the unique solution of the problem (3.6)-(3.10) on the interval $\left[0, T_{0}\right]$.

Theorem 3.3 Let $0<l<1, \Omega \subset \mathbb{R}^{N}$ be a domain with $C^{l+2}$ boundary $\partial \Omega$. Given an initial value $\left(u_{0}, w_{0}\right) \in\left(C^{l+2}(\bar{\Omega})\right)^{2}, u_{0} \geqslant 0, w_{0}>0$ and if the compatibility condition $\frac{\partial u_{0}}{\partial \eta}=u_{0} \frac{\partial w_{0}}{\partial \eta}$ is satisfied for every $x \in \partial \Omega$, then the problem (3.1)-(3.5) has a unique nonnegative solution $(u, w)$ defined on an interval $[0, T) \subset \mathbb{R}$ and $(u, w) \in\left(C^{l+2, l / 2+1}\left(\bar{\Omega}_{t}\right)\right)^{2}$, for all $t \in[0, T)$.

Proof. Theorem 3.2 implies the existence and the uniqueness of the solution of the problem (3.1)-(3.5) on $\bar{\Omega} \times\left[0, \tau_{1}\right]$ with $\tau_{1}$ sufficiently small. By iterating the argument above, we can extend this solution on an interval $\left[\tau_{1}, \tau_{2}\right]$ and so on. At each step the conditions $(i)$ and $(i i)$ in Theorem 3.2 must be fulfilled and this imposes restrictions on the length of the interval of existence. We emphasize that this length depends continuously on the initial data, fact that will be used in the next section for proving the global existence in time of the solution. We obtain in such a way a solution defined in an interval $[0, T) \subset \mathbb{R}$, $0<T \leqslant \infty$. The nonnegativity of the solution results from the maximum principle.

In order to prove the uniqueness of the solution, it is enough to notice that each classical solution of the problem (3.1)-(3.5) can be regarded, locally, as a fixed point of a map analogue to $R \circ S$. The uniqueness of such a fixed point implies the uniqueness of the solution.

\section{Global existence in time}

In this Section we prove that the smooth solution of the problem (3.1)-(3.5) considered in a bounded domain $\Omega \subset \mathbb{R}^{2}$ is globally defined in time. In order to do this, first we derive some a priori estimates which then enable us to prove uniform upper-bound for $|u|_{\Omega_{T}}^{(l+2)}$. Hereafter, $T$ denotes the maximal existence time of the classical nonnegative solution $(u, w)$ to (3.1)-(3.5) obtained in Section 3 corresponding to initial value $\left(u_{0}, w_{0}\right) \in\left(C^{l+2}(\bar{\Omega})\right)^{2}$.

The main result of this Section is:

Theorem 4.1 Let $0<l<1$, and $\Omega \subset \mathbb{R}^{2}$ be a domain with $C^{l+2}$ boundary $\partial \Omega$. Given an initial pair of functions $\left(u_{0}, w_{0}\right) \in\left(C^{l+2}(\bar{\Omega})\right)^{2}$, there exists a global in time nonnegative solution $(u, w) \in$ $\left(C^{l+2, l / 2+1}(\overline{\Omega \times[0, \infty[})\right)^{2}$ to the problem (3.1)-(3.5).

We start by calculating a priori bounds that will be used for proving that the solution $(u, w)$ to the system (3.1)-(3.5) belongs to a suitable Hölder space.

The regularity is then successively ameliorated until obtaining a uniform bound of $|u(\cdot, t)|_{\Omega}^{(l+2)}$ by respect to $t$. As the length of the existence interval obtained in Theorem 3.1 depends uniformly on $\left|u_{0}\right|_{\Omega}^{(l+2)}$, this bound will imply that the maximal interval of definition of the solution is $[0, \infty)$.

In what follows, sometimes the function arguments are omitted and for simplicity we denote with $f_{t}$ the $t$-derivative of the function $f$. Also, the variable $t$ belongs to the maximal time interval of existence of the classical solution $(u, v)$ of the problem (3.1)-(3.5). 


\subsection{A Lyapunov function for the system}

The results obtained in this Subsection do not depend on the dimension of the space, they are valid in a bounded domain $\Omega \subset \mathbb{R}^{N}, N \geqslant 1$.

Proposition 4.2 Suppose that $\left\|u_{0}\right\|_{L^{1}(\Omega)}<\infty$. Then the total mass of the solution $u$ is bounded

$$
\int_{\Omega} u(x, t) d x \leqslant|\Omega| \max \left\{1, M_{0}\right\}
$$

for all $t>0$, where $M_{0}=|\Omega|^{-1}\left\|u_{0}\right\|_{L^{1}(\Omega)}$ represents the initial mass and $|\Omega|$ denotes the volume of $\Omega$.

Proof. Taking into account the boundary condition (3.3) and integrating the equation (3.1) over $\Omega$, we can easily deduce

$$
\int_{\Omega} u_{t}(x, t) d x=\delta \int_{\Omega} u(x, t) d x-\delta \int_{\Omega} u^{2}(x, t) d x .
$$

Applying Jensen's inequality and Gronwall lemma we obtain the estimation (4.1).

Remark 4.1 1. Since the solution $u$ is nonnegative, a consequence of the property (4.1) is that u satisfies an a priori $L^{1}$ estimate uniform in time

$$
\|u\|_{L^{\infty}\left(0, t ; L^{1}(\Omega)\right)}=\left(\left\|u^{1 / 2}\right\|_{L^{\infty}\left(0, t ; L^{2}(\Omega)\right)}\right)^{2} \leqslant|\Omega| \max \left\{1, M_{0}\right\}
$$

for all $t>0$.

2. Let us observe that, from (3.2), we have

$$
w=w_{0} e^{-\int_{0}^{t} u\left[w_{0}^{1-\beta}+(\beta-1) \int_{0}^{s} u\right]^{-1} d s} .
$$

For $w_{0}(x)>0, x \in \Omega$, we obtain $0<w(x, t) \leqslant w_{0}(x)$ for all $t>0$, which implies

$$
\|w\|_{L^{\infty}\left(0, t ; L^{\infty}(\Omega)\right)} \leqslant\left\|w_{0}\right\|_{L^{\infty}(\Omega)} .
$$

We introduce the following two functionals

$$
\begin{aligned}
& F(u, w)=\int_{\Omega} u[\ln u-1] d x+\frac{1}{2} \int_{\Omega} w^{-\beta}|\nabla w|^{2} d x, \\
& D(u, w)=4 \int_{\Omega}\left|\nabla u^{1 / 2}\right|^{2} d x+\frac{\beta}{2} \int_{\Omega} u w^{-1}|\nabla w|^{2} d x+\delta \int_{\Omega} u(u-1) \ln u d x
\end{aligned}
$$

and we show that $F(u, w)$ is a Lyapunov functional to the system (3.1)-(3.5).

Lemma 4.3 If $(u, w)$ is a solutions to the system (3.1)-(3.5), then we have

$$
\frac{d}{d t} F(u, w)=-D(u, w) \leqslant 0 .
$$

Proof. We formally differentiate the functional $F$ with respect to $t$ :

$$
\frac{d}{d t} F(u, w)=\int_{\Omega} u_{t}[\ln u-1] d x+\int_{\Omega} u_{t} d x+\frac{1}{2} \frac{d}{d t} \int_{\Omega} w^{-\beta}|\nabla w|^{2} d x .
$$


Multiplying the equation (3.1) by $\ln u$ and formally integrating on $\Omega$ (in fact we multiply by $\ln (u+\varepsilon$ ), $\varepsilon>0$ and after integration we make $\varepsilon \rightarrow 0$ ), we get

$$
\int_{\Omega} u_{t}[\ln u-1] d x=\int_{\Omega} \Delta u[\ln u-1] d x-\int_{\Omega} \nabla \cdot(u \nabla w)[\ln u-1] d x+\delta \int_{\Omega} u(1-u)[\ln u-1] d x
$$

and taking into account the equality (4.2), we have

$$
\int_{\Omega} u_{t}[\ln u-1] d x=-4 \int_{\Omega}\left|\nabla u^{1 / 2}\right|^{2} d x+\int_{\Omega} \nabla u \cdot \nabla w d x+\delta \int_{\Omega} u(1-u) \ln u d x-\int_{\Omega} u_{t} d x .
$$

Estimating the second term from the right-hand side in the last equality using (3.2):

$$
\int_{\Omega} \nabla u \cdot \nabla w d x=-\frac{1}{2} \frac{d}{d t} \int_{\Omega}\left(w^{-\beta}|\nabla w|^{2}\right) d x-\frac{\beta}{2} \int_{\Omega} u w^{-1}|\nabla w|^{2} d x
$$

and introducing it in (4.8), we obtain (4.7).

Throughout this paper we consider the following assumption on the initial data:

$(\mathcal{H})$ the functions $u_{0}(x) \geqslant 0$ and $w_{0}(x)>0$ satisfy $F\left(u_{0}, w_{0}\right)<+\infty$, for all $x \in \Omega$.

Remark 4.2 1. Let us observe that if the hypothesis $(\mathcal{H})$ is satisfied, then $u_{0} \in L^{1}(\Omega)$ because

$$
\left\|u_{0}\right\|_{L^{1}(\Omega)} \leqslant \int_{\Omega}\left[u_{0}\left(\ln u_{0}-1\right)+e\right] d x \leqslant F\left(u_{0}, w_{0}\right)+e|\Omega| .
$$

2. In fact, because $u(\ln u-1) \geqslant-1$ for all $u>0$, the hypothesis $(\mathcal{H})$ is equivalent with the boundedness of $\int_{\Omega} u_{0} \ln u_{0} d x$ and $w_{0}^{-\frac{\beta-2}{2}} \in H^{1}(\Omega)$ if $\beta \neq 2$ or $\ln w_{0} \in H^{1}(\Omega)$ if $\beta=2$.

Lemma 4.4 If the hypothesis $(\mathcal{H})$ is satisfied, then the functional $F$ is bounded, i.e. there exists a positive constant $C_{4}$ independent on $t$, such that

$$
|F(u, w)| \leqslant C_{4}
$$

for all $t>0$. Moreover, the boundedness independently on $t$ of both terms of the functional $F$ follows.

Proof. Integrating (4.7) between 0 and $t$, we obtain

$$
F(u, w) \leqslant F\left(u_{0}, w_{0}\right)
$$

Let us observe that for all $u>0, u(\ln u-1)>-1$ holds and we have

$$
F(u, w)=\int_{\Omega} u[\ln u-1] d x+\frac{1}{2} \int_{\Omega} w^{-\beta}|\nabla w|^{2} d x \geqslant-|\Omega| .
$$

From (4.11), (4.12) and taking into account also the hypothesis $(\mathcal{H})$ we conclude the lemma with $C_{4}=$ $\max \left\{|\Omega|,\left|F\left(u_{0}, w_{0}\right)\right|\right\}$.

Proposition 4.5 If the hypothesis $(\mathcal{H})$ is satisfied, then there exists a positive constant $C_{5}$ independent on $t$ such that

$$
\int_{\Omega} u \ln u d x<C_{5}
$$

where $C_{5}=C_{5}\left(\int_{\Omega} u_{0} \ln u_{0} d x,\left\|w_{0}\right\|_{H^{1}(\Omega)}^{-\frac{\beta-2}{2}}\right)$ if $\beta \neq 2$, or $C_{5}=C_{5}\left(\int_{\Omega} u_{0} \ln u_{0},\left\|\ln w_{0}\right\|_{H^{1}(\Omega)}\right)$ if $\beta=2$. 
Proof. Taking into account the estimates (4.1) and (4.11), we have

$$
\int_{\Omega} u \ln u d x \leqslant \int_{\Omega} u[\ln u-1] d x+\frac{1}{2} \int_{\Omega} w^{-\beta}|\nabla w|^{2} d x+\int_{\Omega} u d x \leqslant F\left(u_{0}, w_{0}\right)+|\Omega| \max \left\{1, M_{0}\right\} .
$$

Proposition 4.6 If there exists a positive constant $C$, independent on $t$, such that the positive function u satisfies

$$
\int_{\Omega} u \ln u d x<C
$$

then

$$
\lim _{k \rightarrow \infty}\left\|u_{k}\right\|_{L^{1}(\Omega)}=0
$$

uniformly by respect to $t>0$, where $u_{k}=(u-k)_{+}=\max \{0, u-k\}, k>0$.

Proof. For $k>1$ we obtain

$$
\left\|u_{k}\right\|_{L^{1}(\Omega)} \leqslant \int_{\substack{\Omega \\ u(x)>k}} u d x \leqslant \frac{1}{\ln k} \int_{\substack{\Omega \\ u(x)>k}} u \ln u d x \leqslant \frac{1}{\ln k}\left(\int_{\Omega} u \ln u d x-\int_{\substack{\Omega \\ u(x)<1}} u \ln u d x\right) \leqslant C_{6} \frac{1}{\ln k}
$$

where $C_{6}=\left(C+e^{-1}|\Omega|\right)$. The last inequality implies [4.14).

\section{2 $L^{p}$ a priori estimates, $1<p<\infty$}

In order to obtain the desired $L^{p}$-bound on $u$, we make a change of variables of the form

$$
v(x, t)=u(x, t) e^{-w(x, t)}
$$

The system (3.1)- (3.5) becomes

$$
\begin{array}{ll}
\frac{\partial v}{\partial t}=\Delta v+\nabla v \cdot \nabla w+e^{w} v^{2} w^{\beta}+\delta v\left(1-v e^{w}\right) & x \in \Omega, \quad t \in \mathbb{R}_{+} \\
\frac{\partial w}{\partial t}=-e^{w} w^{\beta} v & x \in \Omega, \quad t \in \mathbb{R}_{+} \\
\frac{\partial v}{\partial \eta}=0 & x \in \partial \Omega, \quad t \in \mathbb{R}_{+} \\
v(x, 0)=u_{0}(x) e^{-w_{0}(x)}=v_{0}(x) \geqslant 0 & x \in \Omega \\
w(x, 0)=w_{0}(x)>0 & x \in \Omega
\end{array}
$$

where $\delta \geqslant 0$ and $\beta \geqslant 1$.

Remark 4.3 We shall use this change of variables and the new system (4.16)-(4.20) in order to prove an uniform upper-bound for $|v|_{\Omega_{T}}^{(l+2)}$ and subsequently to establish an uniform upper-bound for $|u|_{\Omega_{T}}^{(l+2)}$.

From now on, for simplicity of notation we shall write $v_{k}$ instead of $(v-k)_{+}$, where $k>0$.

Proposition 4.7 Let $\Omega \subset \mathbb{R}^{2}$ be a bounded domain with smooth boundary. If the hypothesis $(\mathcal{H})$ is satisfied and $v_{0} \in L^{p}(\Omega), 1<p<\infty, w_{0} \in L^{\infty}(\Omega)$, then there exists a constant $C_{7}=C_{7}\left(p,\left\|v_{0}\right\|_{L^{p}(\Omega)},\left\|w_{0}\right\|_{L^{\infty}(\Omega)}\right)$ independent on time such that the solution $v$ to the system (4.16)-4.20) satisfies

$$
\|v\|_{L^{\infty}\left(0, T ; L^{p}(\Omega)\right)} \leqslant C_{7}, \quad \forall 1 \leqslant p<+\infty .
$$


Proof. Testing the equation (4.16) with $p v_{k}^{p-1} e^{w}, k>0, p>1$, gives

$$
\frac{d}{d t} \int_{\Omega} v_{k}^{p} e^{w}=-p(p-1) \int_{\Omega} v_{k}^{p-2} e^{w}\left|\nabla v_{k}\right|^{2}+\delta p \int_{\Omega} v_{k}^{p-1}\left[v e^{w}\left(1-v e^{w}\right)\right]+(p-1) \int_{\Omega} e^{2 w} w^{\beta} v_{k}^{p+1} .
$$

Taking into account the identity

$$
\left|\nabla\left(v_{k}^{p / 2}\right)\right|^{2}=\frac{p^{2}}{4} v_{k}^{p-2}\left|\nabla v_{k}\right|^{2}
$$

we obtain from (4.22)

$$
\begin{aligned}
\frac{d}{d t} \int_{\Omega} v_{k}^{p} e^{w} & =-\frac{4(p-1)}{p} \int_{\Omega} e^{w}\left|\nabla\left(v_{k}^{p / 2}\right)\right|^{2}+\int_{\Omega} p k e^{w}\left[k e^{w} w^{\beta}+\delta\left(1-k e^{w}\right)\right] v_{k}^{p-1}+ \\
& +\int_{\Omega} e^{w}\left[(2 p-1) k e^{w} w^{\beta}+\delta p\left(1-2 k e^{w}\right)\right] v_{k}^{p}+\int_{\Omega} e^{2 w}\left[(p-1) w^{\beta}-\delta p\right] v_{k}^{p+1} .
\end{aligned}
$$

Since $0<w(x, t) \leqslant w_{0}(x)$ and $e^{w(x, t)} \geqslant 1$ for all $x \in \Omega, t>0$, we obtain from (4.23)

$$
\frac{d}{d t} \int_{\Omega} v_{k}^{p} e^{w} \leqslant-\frac{4(p-1)}{p}\left\|\nabla\left(v_{k}^{p / 2}\right)\right\|_{L^{2}(\Omega)}^{2}+C_{8} \int_{\Omega} v_{k}^{p-1}+C_{9} \int_{\Omega} v_{k}^{p}+C_{10} \int_{\Omega} v_{k}^{p+1}
$$

where we have made the following notations

$$
\begin{aligned}
& C_{8}=C_{8}\left(p, k, \delta,\left\|w_{0}\right\|_{L^{\infty}(\Omega)}\right)=
\end{aligned}
$$

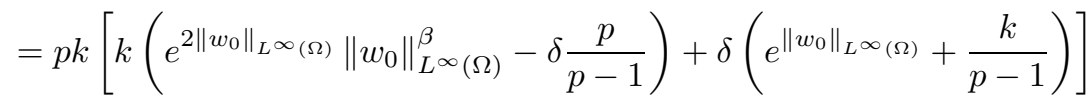

$$
\begin{aligned}
& C_{9}=C_{9}\left(p, k, \delta,\left\|w_{0}\right\|_{L^{\infty}(\Omega)}\right)= \\
& =p\left[\frac{2 p-1}{p} k\left(e^{2\left\|w_{0}\right\|_{L^{\infty}(\Omega)}}\left\|w_{0}\right\|_{L^{\infty}(\Omega)}^{\beta}-\delta \frac{p}{p-1}\right)+\delta\left(e^{\left\|w_{0}\right\|_{L^{\infty}(\Omega)}}+\frac{k}{p-1}\right)\right]
\end{aligned}
$$

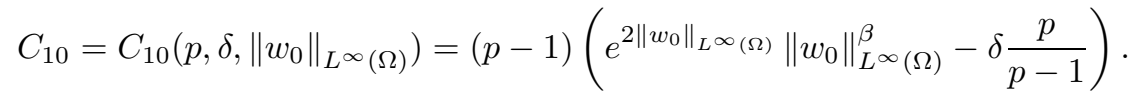

Adding the term $\sigma \int_{\Omega} v_{k}^{p}$, where $\sigma>0$ is a constant, on both sides of the last inequality, we obtain

$$
\frac{d}{d t} \int_{\Omega} v_{k}^{p} e^{w}+\sigma \int_{\Omega} v_{k}^{p} \leqslant-\frac{4(p-1)}{p}\left\|\nabla\left(v_{k}^{p / 2}\right)\right\|_{L^{2}(\Omega)}^{2}+C_{8} \int_{\Omega} v_{k}^{p-1}+\left(C_{9}+\sigma\right) \int_{\Omega} v_{k}^{p}+C_{10} \int_{\Omega} v_{k}^{p+1} .
$$

We estimate now the last two terms from (4.28) using Gagliardo-Nirenberg's inequality and taking into account the positivity of $v$. We have

$$
\begin{aligned}
& \int_{\Omega} v_{k}^{p}=\left\|v_{k}^{\frac{p}{2}}\right\|_{L^{2}(\Omega)}^{2} \leqslant C_{11}(\Omega)\left\|v_{k}^{\frac{p}{2}}\right\|_{H^{1}(\Omega)}\left\|v_{k}^{\frac{p}{2}}\right\|_{L^{1}(\Omega)}, \\
& \int_{\Omega} v_{k}^{p+1}=\left\|v_{k}^{\frac{p}{2}}\right\|_{L^{\frac{2(p+1)}{p}}}^{\frac{2(p+1)}{p}(\Omega)} \leqslant C_{12}(\Omega)\left\|v_{k}^{\frac{p}{2}}\right\|_{H^{1}(\Omega)}^{2}\left\|v_{k}\right\|_{L^{1}(\Omega)} .
\end{aligned}
$$


We insert the estimations (4.29), (4.30) into (4.28) and we apply Cauchy's inequality. We obtain

$$
\begin{aligned}
\frac{d}{d t} \int_{\Omega} v_{k}^{p} e^{w}+\sigma \int_{\Omega} v_{k}^{p} & \leqslant-\frac{4(p-1)}{p}\left\|\nabla\left(v_{k}^{\frac{p}{2}}\right)\right\|_{L^{2}(\Omega)}^{2}+C_{8}\left\|v_{k}^{p-1}\right\|_{L^{1}(\Omega)}+ \\
& +\left[C_{9}+\sigma\right]\left\|v_{k}^{\frac{p}{2}}\right\|_{L^{2}(\Omega)}^{2}+C_{10} C_{12}\left\|v_{k}^{\frac{p}{2}}\right\|_{H^{1}(\Omega)}^{2}\left\|v_{k}\right\|_{L^{1}(\Omega)} \leqslant \\
& \leqslant\left[-\frac{4(p-1)}{p}+C_{10} C_{12}\left\|v_{k}\right\|_{L^{1}(\Omega)}+\varepsilon\right]\left\|\nabla\left(v_{k}^{\frac{p}{2}}\right)\right\|_{L^{2}(\Omega)}^{2}+C_{8}\left\|v_{k}^{p-1}\right\|_{L^{1}(\Omega)}+ \\
& +\varepsilon\left\|v_{k}^{\frac{p}{2}}\right\|_{L^{2}(\Omega)}^{2}+\frac{1}{4 \varepsilon}\left\{C_{11}\left[C_{9}+\sigma+C_{10} C_{12}\left\|v_{k}\right\|_{L^{1}(\Omega)}\right]\right\}^{2}\left\|v_{k}^{\frac{p}{2}}\right\|_{L^{1}(\Omega)}^{2} .
\end{aligned}
$$

In order to estimate the second term from the right-hand side of (4.31), we apply Young's inequality and we obtain for $\epsilon>0$

$$
v_{k}^{p-1} \leqslant \frac{1}{p} \epsilon^{-p}+\frac{p-1}{p} \epsilon^{\frac{p}{p-1}} v_{k}^{p} .
$$

Now, choosing $\varepsilon$ small enough such that $\varepsilon<\min \{\sigma / 2,2(p-1) / p\}$ and inserting (4.32) in (4.31), we get

$$
\begin{aligned}
\frac{d}{d t} \int_{\Omega} v_{k}^{p} e^{w}+\frac{\sigma}{2} \int_{\Omega} v_{k}^{p} & \leqslant\left[-\frac{4(p-1)}{p}+C_{10} C_{12}\left\|v_{k}\right\|_{L^{1}(\Omega)}+\varepsilon\right]\left\|\nabla\left(v_{k}^{\frac{p}{2}}\right)\right\|_{L^{2}(\Omega)}^{2}+\frac{1}{p} \epsilon^{-p} C_{8}|\Omega|+ \\
& +\frac{1}{4 \varepsilon}\left\{C_{11}\left[C_{9}+\sigma+\frac{p-1}{p} \epsilon^{\frac{p}{p-1}} C_{8}+C_{10} C_{12}\left\|v_{k}\right\|_{L^{1}(\Omega)}\right]\right\}^{2}\left\|v_{k}^{\frac{p}{2}}\right\|_{L^{1}(\Omega)}^{2} .
\end{aligned}
$$

Taking into account Proposition 4.6, we can choose $k$ sufficiently large such that the coefficient of $\left\|\nabla\left(v_{k}^{\frac{p}{2}}\right)\right\|_{L^{2}(\Omega)}^{2}$ is negative. In this way, using (4.4), the last inequality becomes

$$
\frac{d}{d t} \int_{\Omega} v_{k}^{p} e^{w}+\frac{\sigma}{2 e^{\left\|w_{0}\right\|_{L^{\infty}(\Omega)}}} \int_{\Omega} v_{k}^{p} e^{w} \leqslant C_{13}\left\|v_{k}^{\frac{p}{2}}\right\|_{L^{1}(\Omega)}^{2}+\frac{1}{p} \epsilon^{-p} C_{8}|\Omega|
$$

where

$$
C_{13}=C_{13}\left(p, k, \delta,\left\|w_{0}\right\|_{L^{\infty}(\Omega)}, \sigma, \varepsilon, \epsilon, \Omega\right)=\frac{1}{4 \varepsilon}\left\{C_{11}\left[C_{9}+\sigma+\frac{p-1}{p} \epsilon^{\frac{p}{p-1}} C_{8}+C_{10} C_{12}\left\|v_{k}\right\|_{L^{1}(\Omega)}\right]\right\}^{2} .
$$

Applying Gronwall's inequality, we obtain from (4.33)

$$
\int_{\Omega} v_{k}^{p} \leqslant \int_{\Omega} v_{k}^{p} e^{w} \leqslant \max \left\{\int_{\Omega}\left(v_{0}-k\right)_{+}^{p} e^{w_{0}}, \frac{2 e^{\left\|w_{0}\right\|_{L^{\infty}(\Omega)}}}{\sigma}\left[C_{13}\left\|v_{k}^{\frac{p}{2}}\right\|_{L^{1}(\Omega)}^{2}+\frac{1}{p} \epsilon^{-p} C_{8}|\Omega|\right]\right\} .
$$

We will show by induction that

$$
\left\|v_{k}(t)\right\|_{L^{p}(\Omega)} \leqslant C
$$

for all $p=2^{j}$, with $j \in \mathbb{N}$, where $C$ is a constant independent of $t$.

Let us remark that, taking into account Proposition 4.2, we have

$$
\left\|v_{k}(t)\right\|_{L^{1}(\Omega)} \leqslant|\Omega| \max \left\{1, M_{0}\right\} .
$$

Let $p=2^{j}$, and suppose that $\left\|v_{k}(t)\right\|_{L^{2^{j-1}}(\Omega)}=\left\|v_{k}(t)\right\|_{L^{p / 2}(\Omega)}$ is uniformly bounded, the bound being independent of $t>0$. We obtain from (4.34) that $\left\|v_{k}(t)\right\|_{L^{2^{j}(\Omega)}}$ is bounded, $j \in \mathbb{N} \backslash\{0\}$. We conclude, taking into account the embeddings of $L^{p}(\Omega)$ spaces, that

$$
\left\|v_{k}\right\|_{L^{\infty}\left(0, t ; L^{p}(\Omega)\right)} \leqslant C_{14}, \text { for every } 1 \leqslant p<\infty
$$


where $C_{14}=C_{14}\left(p,\left\|v_{0}\right\|_{L^{1}(\Omega)},\left\|w_{0}\right\|_{L^{\infty}(\Omega)}\right)$ is a positive constant, independent of $t>0$.

Finally, we obtain

$$
\|v\|_{L^{p}(\Omega)} \leqslant 2\left(\left\|v_{k}(t)\right\|_{L^{p}(\Omega)}^{p}+k^{p}|\Omega|\right)^{1 / p}
$$

and we conclude the Theorem.

Remark 4.4 The above estimations are strongly dependent on the dimension of the space and they are done in the case when $C_{8}, C_{9}, C_{10}$ are positive. If one or several of these constants are negative (for

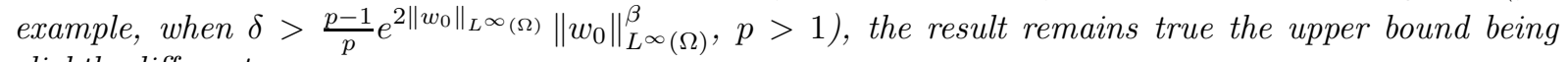
slightly different.

\section{3 $\quad L^{\infty}$ a priori estimates}

Proposition 4.8 Let $\Omega \subset \mathbb{R}^{2}$ be a bounded domain with smooth boundary. If the hypothesis $(\mathcal{H})$ is satisfied, $v_{0} \in L^{\infty}(\Omega)$ and $w_{0} \in L^{\infty}(\Omega)$, then the solution $v$ of the system (4.16)-(4.20) satisfies

$$
\|v\|_{L^{\infty}\left(0, T ; L^{\infty}(\Omega)\right)} \leqslant C
$$

where $C$ is a positive constant independent on time which will be determined later.

Proof. We introduce the following sets

$$
\Omega_{k}(t)=\{x \in \Omega ; \quad v(x, t)>k\}
$$

where $k$ is a positive constant. Let us observe that, taking into account (4.35) and choosing $p=2$, the relation (4.31) becomes

$$
\begin{aligned}
\frac{d}{d t} \int_{\Omega} v_{k}^{2} e^{w}+\sigma \int_{\Omega} v_{k}^{2} & \leqslant\left[-2+C_{10} C_{12}\left\|v_{k}\right\|_{L^{1}(\Omega)}+\varepsilon\right]\left\|\nabla v_{k}\right\|_{L^{2}(\Omega)}^{2}+\varepsilon\left\|v_{k}\right\|_{L^{2}(\Omega)}^{2}+ \\
& +\left\{C_{8}+\frac{1}{4 \varepsilon}\left\|v_{k}\right\|_{L^{1}(\Omega)}\left[C_{11}\left(C_{9}+\sigma+C_{10} C_{12}\left\|v_{k}\right\|_{L^{1}(\Omega)}\right)\right]^{2}\right\}\left\|v_{k}\right\|_{L^{1}(\Omega)} .
\end{aligned}
$$

We estimate the last term of the right-hand side of the last inequality using Hölder's inequality and a Sobolev embedding

$$
\left\|v_{k}\right\|_{L^{1}(\Omega)} \leqslant\left\|v_{k}\right\|_{L^{4}(\Omega)}\left|\Omega_{k}\right|^{3 / 4} \leqslant C_{15}\left\|v_{k}\right\|_{H^{1}(\Omega)}\left|\Omega_{k}\right|^{3 / 4}
$$

where $C_{15}$ is a constant independent of $t$. Using this inequality and Cauchy's inequality, we obtain from (4.36)

$$
\begin{aligned}
\frac{d}{d t} \int_{\Omega} v_{k}^{2} e^{w}+\sigma \int_{\Omega} v_{k}^{2} & \leqslant\left[-2+C_{10} C_{12}\left\|v_{k}\right\|_{L^{1}(\Omega)}+\varepsilon+\varepsilon^{\prime}\right]\left\|\nabla v_{k}\right\|_{L^{2}(\Omega)}^{2}+\left(\varepsilon+\varepsilon^{\prime}\right)\left\|v_{k}\right\|_{L^{2}(\Omega)}^{2}+ \\
& +\frac{C_{15}^{2}}{4 \varepsilon^{\prime}}\left\{C_{8}+\frac{1}{4 \varepsilon}\left\|v_{k}\right\|_{L^{1}(\Omega)}\left[C_{11}\left(C_{9}+\sigma+C_{10} C_{12}\left\|v_{k}\right\|_{L^{1}(\Omega)}\right)\right]^{2}\right\}^{2}\left|\Omega_{k}\right|^{3 / 2} .
\end{aligned}
$$

We choose $\varepsilon$ and $\varepsilon^{\prime}$ small enough such that $\varepsilon+\varepsilon^{\prime}<\min \{1, \sigma / 2\}$. Taking into account Proposition 4.6, it follows that there exists $k_{1}>0$ sufficiently large such that, for every $k>k_{1}$, the coefficient of $\left\|\nabla v_{k}\right\|_{L^{2}(\Omega)}^{2}$ is negative. Taking into account (4.4) and (4.35) we obtain from (4.37)

$$
\frac{d}{d t} \int_{\Omega} v_{k}^{2} e^{w}+\frac{\sigma}{2 e^{\left\|w_{0}\right\|_{L^{\infty}(\Omega)}}} \int_{\Omega} v_{k}^{2} e^{w} \leqslant C_{16}\left|\Omega_{k}\right|^{3 / 2}
$$


for all $k>k_{1}$, where

$$
\begin{aligned}
C_{16} & =C_{16}\left(k, \delta,\left\|w_{0}\right\|_{L^{\infty}(\Omega)}, M_{0}\right)= \\
& =\frac{C_{15}^{2}}{4 \varepsilon^{\prime}}\left\{C_{8}+\frac{1}{4 \varepsilon}|\Omega| \max \left\{1, M_{0}\right\}\left[C_{11}\left(C_{9}+\sigma+C_{10} C_{12}|\Omega| \max \left\{1, M_{0}\right\}\right)\right]^{2}\right\}^{2} .
\end{aligned}
$$

One can notice, using (4.25), (4.26) and (4.27), that $C_{16}$ is a polynomial of degree 4 in $k$. In the first place we shall focus on obtaining an inequality similar to (4.38) where the constant appearing in the right-hand side is independent on $k$. Let $\alpha$ be the dominant coefficient of $C_{16}$ as a polynomial in $k$. It is a constant depending only on the initial data of the system. On the other hand, we have (see [26])

$$
\int_{\Omega} v^{q+1}=(q+1) \int_{0}^{\infty} s^{q}\left|\Omega_{s}\right| d s, \quad q \geqslant 1 .
$$

We obtain, using these facts, a bound for the right-hand side of the inequality (4.38). Namely, taking into account Proposition 4.7, we get first

$$
(k-1)^{q}\left|\Omega_{k}\right|<\int_{k-1}^{k} s^{q}\left|\Omega_{s}\right| d s<\int_{0}^{\infty} s^{q}\left|\Omega_{s}\right| d s=\frac{1}{q+1}\|v\|_{L^{q+1}(\Omega)}^{q+1}<C_{17}
$$

where $C_{17}$ is a constant independent on $k$ and on $t$. From the last inequality, taking $q=16$, we obtain

$$
(k-1)^{4}\left|\Omega_{k}\right|^{1 / 4}<C_{17}^{1 / 4}
$$

It follows that there exists $k_{2}>0$ such that for every $k>k_{2}$,

$$
C_{16}\left|\Omega_{k}\right|^{1 / 4}<(\alpha+1) C_{17}^{1 / 4}=C_{18}
$$

which implies, from (4.38)

$$
\frac{d}{d t} \int_{\Omega} v_{k}^{2} e^{w}+\frac{\sigma}{2 e^{\left\|w_{0}\right\|_{L} \infty(\Omega)}} \int_{\Omega} v_{k}^{2} e^{w} \leqslant C_{18}\left|\Omega_{k}\right|^{5 / 4}
$$

In this way we obtained an inequality similar to (4.38) where the constant $C_{18}$ does not depend on $k$. Since $v_{0} \in L^{\infty}(\Omega)$, there exists $k_{3}>0$ such that $\left\|v_{k}(0)\right\|_{L^{\infty}(\Omega)}=0$ for all $k>k_{3}$. For $k>\max \left\{k_{1}, k_{2}, k_{3}\right\}$, we deduce from (4.39) using Gronwall's inequality

$$
\left\|v_{k}(t)\right\|_{L^{2}(\Omega)}^{2} \leqslant\left\|e^{w / 2} v_{k}(t)\right\|_{L^{2}(\Omega)}^{2} \leqslant \frac{2 C_{18} e^{\left\|w_{0}\right\|_{L^{\infty}(\Omega)}}}{\sigma}\left(1-e^{-\frac{\sigma}{2 e^{\left\|w_{0}\right\|_{L} \infty(\Omega)}} t}\right)\left(\sup _{t \geqslant 0}\left|\Omega_{k}(t)\right|\right)^{5 / 4} .
$$

On the other hand, taking into account that $\Omega_{l} \subset \Omega_{k}$ for $l>k>0$,

$$
\left\|v_{k}(t)\right\|_{L^{2}(\Omega)}^{2} \geqslant \int_{\Omega_{l}(t)} v_{k}^{2} \geqslant(l-k)^{2}\left|\Omega_{l}(t)\right|
$$

Taking the supremum on $t \geqslant 0$ in the last relation, (4.40) implies

$$
(l-k)^{2} \sup _{t \geqslant 0}\left|\Omega_{l}(t)\right| \leqslant \frac{2 C_{18} e^{\left\|w_{0}\right\|_{L \infty}(\Omega)}}{\sigma}\left(\sup _{t \geqslant 0}\left|\Omega_{k}(t)\right|\right)^{5 / 4}
$$


for $l>k>\max \left\{k_{1}, k_{2}, k_{3}\right\}$. Obviously the function $k \mapsto \sup _{t \geqslant 0}\left|\Omega_{k}(t)\right|$ is decreasing, so we can apply Lemma 4.1 from [8]. It follows that there exists

$$
k_{0}=\max \left\{k_{1}, k_{2}, k_{3}\right\}+\left(\frac{2^{11} C_{18} e^{\left\|w_{0}\right\|_{L^{\infty}(\Omega)}}}{\sigma}\right)^{1 / 2}|\Omega|^{1 / 8}
$$

such that

$$
\sup _{t \geqslant 0}\left|\Omega_{k}(t)\right|=0
$$

for all $k \geqslant k_{0}$. This concludes the proof.

Remark 4.5 The $L^{\infty}$ bound can also be proved using the iterative technique of Alikakos [1]. We have chosen the method presented here (inspired by an idea of Gajewski and Zacharias [11]) mainly for aesthetic reasons.

It is obvious that the conclusion of Proposition 4.8 remains valid also in the case of the classical solution $u$ of the system (3.1)-(3.5).

\subsection{A priori estimates for $\nabla v, \nabla w$ and $\Delta v$}

Taking the initial data in $\left(W^{2, q}\right)^{2}, q>2$, in [13] the authors derive $L^{\infty}\left(0, t ; L^{p}(\Omega)\right), 1<p \leqslant q$ bounds for $\nabla v, \nabla w$ and $\Delta v$. Based on these estimates, under $L^{\infty}$ bounds assumptions, they show the global existence of weak solutions. Moreover, under the same hypotheses on the initial data, it is proved that the solution has some regularity properties.

By a different strategy we establish hereafter a priori bounds for $\|\nabla v\|_{L^{1}\left(0, t ; L^{p}(\Omega)\right)},\|\Delta v\|_{L^{1}\left(0, t ; L^{2}(\Omega)\right)}$ and $\sup \|\nabla w\|_{L^{p}(\Omega)}, p \geqslant 2$. We mention that both lines of computation could be applied, as an intermediary $t \in[0, T]$

step, in order to obtain classical solutions. However, using the a priori bounds which are given in what follows, one may prove the existence of the weak solutions (in the sense of [13]) of the problem (3.1)-(3.5) starting with the initial data in, for example, $\left(H^{1}(\Omega) \cap L^{\infty}(\Omega)\right) \times W^{1,4}(\Omega)$.

Lemma 4.9 Let $\Omega \subset \mathbb{R}^{2}$ be a bounded domain. If the hypothesis $(\mathcal{H})$ is satisfied, $v_{0} \in H^{1}(\Omega) \cap L^{\infty}(\Omega)$ and $w_{0} \in L^{\infty}(\Omega)$, we have

$$
\begin{aligned}
\left\|v_{t}\right\|_{L^{2}\left(\Omega_{T}\right)} & \leqslant C_{20} \\
\|\nabla v\|_{L^{2}(\Omega)} & \leqslant C_{20}
\end{aligned}
$$

for all $t>0$, where $C_{20}$ is a constant independent on $t$.

Proof. Taking $e^{w} v_{t}$ as a test function in the equation (4.16) and integrating in space, we obtain

$$
\int_{\Omega} e^{w} v_{t}^{2}+\frac{1}{2} \frac{d}{d t} \int_{\Omega}\left(e^{w}|\nabla v|^{2}\right)=-\frac{1}{2} \int_{\Omega} e^{2 w} v w^{\beta}|\nabla v|^{2}+\int_{\Omega} e^{w}\left[e^{w} v^{2} w^{\beta}+\delta v\left(1-v e^{w}\right)\right] v_{t} .
$$

In order to estimate the last term from the right-hand side of (4.44) we take into account the following inequalities

$$
\begin{aligned}
& \int_{\Omega} e^{2 w} w^{\beta} v^{2} v_{t} \leqslant \frac{1}{2} \int_{\Omega} e^{w} v_{t}^{2}-\frac{1}{2} e^{2\left\|w_{0}\right\|_{L^{\infty}(\Omega)}}\left\|w_{0}\right\|_{L^{\infty}(\Omega)}\|v\|_{L^{\infty}(\Omega)}^{3} \int_{\Omega} \frac{\partial w}{\partial t} \\
& \delta \int_{\Omega} e^{w} v v_{t} \leqslant \frac{\delta}{2} \frac{d}{d t} \int_{\Omega} e^{w} v^{2}-\frac{\delta}{2} e^{\left\|w_{0}\right\|_{L^{\infty}(\Omega)}\|v\|_{L^{\infty}(\Omega)}^{2} \int_{\Omega} \frac{\partial w}{\partial t}} \\
& -\delta \int_{\Omega} e^{2 w} v^{2} v_{t} \leqslant-\frac{\delta}{3} \frac{d}{d t} \int_{\Omega} e^{2 w} v^{3} .
\end{aligned}
$$


Substituting (4.45), (4.46) and (4.47) into (4.44), after that integrating in time and taking into account (4.4) we obtain

$$
\int_{0}^{t} \int_{\Omega} v_{s}^{2}+\int_{\Omega} e^{w}|\nabla v|^{2} \leqslant \int_{\Omega} e^{w_{0}}\left|\nabla v_{0}\right|^{2}+C_{19}
$$

where

$$
\begin{aligned}
C_{19} & =e^{\left\|w_{0}\right\|_{L^{\infty}(\Omega)}}\|v\|_{L^{\infty}\left(0, t ; L^{\infty}(\Omega)\right)}^{2}\left[e^{\left\|w_{0}\right\|_{L^{\infty}(\Omega)}}\left\|w_{0}\right\|_{L^{\infty}(\Omega)}^{\beta}\|v\|_{L^{\infty}\left(0, t ; L^{\infty}(\Omega)\right)}+\delta\right]\left\|w_{0}\right\|_{L^{1}(\Omega)}+ \\
& +\delta e^{\left\|w_{0}\right\|_{L^{\infty}(\Omega)}}\|v\|_{L^{2}(\Omega)}^{2}+\frac{2 \delta}{3} \int_{\Omega} e^{2 w_{0}} v_{0}^{3}
\end{aligned}
$$

The last inequality implies (4.42) and (4.43) where $C_{20}=\left(e^{\left\|w_{0}\right\|_{L} \infty(\Omega)}\left\|\nabla v_{0}\right\|_{L^{2}(\Omega)}^{2}+C_{19}\right)^{1 / 2}$.

Lemma 4.10 Let $\Omega \subset \mathbb{R}^{2}$ be a bounded domain. If the hypothesis $(\mathcal{H})$ is satisfied, $v_{0} \in H^{1}(\Omega) \cap L^{\infty}(\Omega)$ and $w_{0} \in W^{1,4}(\Omega)$, we have

$$
\|\Delta v\|_{L^{1}\left(0, t ; L^{2}(\Omega)\right)} \leqslant e(n+1) ! k\left(T_{0}\right)
$$

for all $t \in\left[0, \min \left\{(n+1) T_{0}, T\right\}\right], n \in \mathbb{N} \backslash\{0\}$, where $T_{0}$ is a constant independent on $t$ and $k$ is a function with liniar growing which will be given later.

Proof. From (4.16) we obtain for every $0 \leqslant t<T$

$$
\int_{0}^{t}\|\Delta v\|_{L^{2}(\Omega)} \leqslant \int_{0}^{t}\left\|v_{s}\right\|_{L^{2}(\Omega)}+\int_{0}^{t}\|\nabla w \cdot \nabla v\|_{L^{2}(\Omega)}+\int_{0}^{t}\|h(v, w)\|_{L^{2}(\Omega)}
$$

where

$$
h(v, w)=e^{w} v^{2} w^{\beta}+\delta v\left(1-v e^{w}\right) .
$$

We estimate the first term from (4.50) using (4.42) and the Hölder inequality

$$
\int_{0}^{t}\left\|v_{s}\right\|_{L^{2}(\Omega)} \leqslant t^{1 / 2}\left(\int_{0}^{t} \int_{\Omega}\left|v_{s}\right|^{2}\right)^{1 / 2} \leqslant \sqrt{C_{20}} t^{1 / 2} .
$$

In order to obtain an estimate for $\|\nabla w\|_{L^{4}(\Omega)}$, we deduce from the equation (4.17)

$$
\nabla w_{t}=-e^{w} w^{\beta} v \nabla w-\beta e^{w} w^{\beta-1} v \nabla w-e^{w} w^{\beta} \nabla v
$$

Multiplying the last relation by $\nabla w|\nabla w|^{2}$ we obtain by integration

$$
\|\nabla w\|_{L^{4}(\Omega)} \leqslant C_{22}+C_{21} \int_{0}^{t}\|\nabla v\|_{L^{4}(\Omega)}
$$

where $C_{21}=e^{\left\|w_{0}\right\|_{L^{\infty}(\Omega)}}\left\|w_{0}\right\|_{L^{\infty}(\Omega)}^{\beta}, C_{22}=\left\|\nabla w_{0}\right\|_{L^{4}(\Omega)}$. Taking into account (4.52) and the Hölder inequality we obtain

$$
\|\nabla w \cdot \nabla v\|_{L^{2}(\Omega)} \leqslant C_{22}\|\nabla v\|_{L^{4}(\Omega)}+\frac{C_{21}}{2} \frac{d}{d t}\left[\int_{\tau}^{t}\|\nabla v\|_{L^{4}(\Omega)}\right]^{2} .
$$


In order to estimate the second term of (4.53), we obtain from the Hölder inequality and (4.43)

$$
\left[\int_{0}^{t}\|\nabla v\|_{L^{4}(\Omega)}\right]^{2} \leqslant C_{23}^{2} t^{1 / 2}\left(\int_{0}^{t}\|\Delta v\|_{L^{2}(\Omega)}\right)\left(\int_{0}^{t} \int_{\Omega}|\nabla v|^{2}\right)^{1 / 2} \leqslant C_{23}^{2} \sqrt{C_{20}} t \int_{0}^{t}\|\Delta v\|_{L^{2}(\Omega)} .
$$

We estimate now the second term from (4.50). Finally, after integration of (4.53) on $[0, t]$, taking into account (4.54) and using the Cauchy inequality and the Gagliardo-Nirenberg inequality in order to estimate the first term of (4.53),

$$
\int_{0}^{t}\|\nabla w \cdot \nabla v\|_{L^{2}(\Omega)} \leqslant \varepsilon \int_{0}^{t}\|\Delta v\|_{L^{2}(\Omega)}+\frac{\left(C_{22} C_{23}\right)^{2}}{4 \varepsilon} \int_{0}^{t}\|\nabla v\|_{L^{2}(\Omega)}+\frac{C_{21} C_{23}^{2} \sqrt{C_{20}}}{2} t \int_{0}^{t}\|\Delta v\|_{L^{2}(\Omega)} .
$$

We estimate the last term from (4.50) using (4.21)

$$
\int_{0}^{t}\|h(v, w)\|_{L^{2}(\Omega)} \leqslant C_{24} t
$$

where $C_{24}=\delta\|v\|_{L^{2}(\Omega)}+e^{\left\|w_{0}\right\|_{L^{\infty}(\Omega)}}\left[\delta+\left\|w_{0}\right\|_{L^{\infty}(\Omega)}^{\beta}\right]\|v\|_{L^{4}(\Omega)}^{2}$.

Taking into account (4.43), (4.51), (4.55) and (4.56) we estimate now $\|\Delta v\|_{L^{2}(\Omega)}^{2}$ from (4.50)

$$
\left(1-\varepsilon-\frac{C_{21} C_{23}^{2} \sqrt{C_{20}}}{2} t\right) \int_{0}^{t}\|\Delta v\|_{L^{2}(\Omega)} \leqslant \sqrt{C_{20}} t^{1 / 2}+t\left[\frac{\left(C_{22} C_{23}\right)^{2}}{4 \varepsilon} \sqrt{C_{20}}+C_{24}\right] .
$$

We take $\varepsilon=\frac{1}{4}$ and $t$ sufficiently small such that

$$
1-\varepsilon-\frac{C_{21} C_{23}^{2} \sqrt{C_{20}}}{2} t \geqslant \frac{1}{2} \Longrightarrow t \leqslant \frac{1}{2 C_{21} C_{23}^{2} \sqrt{C_{20}}}=T_{0}
$$

which implies from (4.57)

$$
\int_{0}^{t}\|\Delta v\|_{L^{2}(\Omega)} \leqslant 2 \sqrt{C_{20}} t^{1 / 2}+2\left[\left(C_{22} C_{23}\right)^{2} \sqrt{C_{20}}+C_{24}\right] t=k(t) .
$$

In this way we have obtained the boundedness for $\int_{0}^{t}\|\Delta v\|_{L^{2}(\Omega)}$ for all $t \in\left[0, \min \left\{T_{0}, T\right\}\right]$. This bound depends on the initial data considered in $\tau=0$.

If $T_{0}<T$ we can repeat the procedure taking the initial data in $\tau=T_{0}$ and in a similar manner we obtain

$$
\begin{aligned}
& \left(1-\varepsilon-\frac{C_{21} C_{23}^{2} \sqrt{C_{20}}}{2}\left(t-T_{0}\right)\right) \int_{T_{0}}^{t}\|\Delta v\|_{L^{2}(\Omega)} \leqslant \sqrt{C_{20}}\left(t-T_{0}\right)^{1 / 2}+ \\
& +\left(t-T_{0}\right)\left\{\frac{C_{23}^{2} \sqrt{C_{20}}}{4 \varepsilon}\left[2 C_{22}^{2}+C_{21} \int_{0}^{T_{0}}\|\Delta v\|_{L^{2}(\Omega)}\right]+C_{24}\right\} .
\end{aligned}
$$

Taking $\varepsilon=\frac{1}{4}$ and $T_{0}<t \leqslant 2 T_{0}$ from (4.59) we have

$$
\int_{T_{0}}^{t}\|\Delta v\|_{L^{2}(\Omega)} \leqslant k\left(t-T_{0}\right)+\frac{1}{T_{0}}\left(t-T_{0}\right) \int_{0}^{T_{0}}\|\Delta v\|_{L^{2}(\Omega)} .
$$


The last relation is true for all $t \in\left[T_{0}, \min \left\{2 T_{0}, T\right\}\right]$. More generally, we obtain

$$
\int_{n T_{0}}^{t}\|\Delta v\|_{L^{2}(\Omega)} \leqslant k\left(t-n T_{0}\right)+\frac{n}{T_{0}}\left(t-n T_{0}\right) \int_{0}^{n T_{0}}\|\Delta v\|_{L^{2}(\Omega)}
$$

for all $t \in\left[n T_{0}, \min \left\{(n+1) T_{0}, T\right\}\right]$, if $n \in \mathbb{N} \backslash\{0\}$ is such that $n T_{0}<T$.

Let us observe that $t-n T_{0} \leqslant T_{0}$ and the function $k(t)$ given by (4.58) is nondecreasing. Thus, the inequality (4.60) becomes

$$
\int_{n T_{0}}^{t}\|\Delta v\|_{L^{2}(\Omega)} \leqslant k\left(T_{0}\right)+n \int_{0}^{n T_{0}}\|\Delta v\|_{L^{2}(\Omega)}
$$

for all $t \in\left[n T_{0}, \min \left\{(n+1) T_{0}, T\right\}\right]$.

Finally, for all $t \in\left[0, \min \left\{(n+1) T_{0}, T\right\}\right]$, taking into account (4.58), we obtain

$$
\begin{aligned}
\int_{0}^{t}\|\Delta v\|_{L^{2}(\Omega)} & \leqslant(n+1) ! k\left(T_{0}\right)\left(\frac{1}{2 !}+\ldots+\frac{1}{(n+1) !}\right)+(n+1) ! \int_{0}^{t}\|\Delta v\|_{L^{2}(\Omega)} \leqslant \\
& \leqslant(n+1) !\left(1+\frac{1}{2 !}+\ldots+\frac{1}{(n+1) !}\right) k\left(T_{0}\right) \leqslant e(n+1) ! k\left(T_{0}\right) .
\end{aligned}
$$

Remark 4.6 The last inequality holds for all $0<t<T$, and $n$ is maximal with the property $n T_{0} \leqslant t$. We emphasize that the bound in terms of $n$ is equivalent with a bound in terms of $t$, of the same type. Hence we obtain

$$
\int_{0}^{t}\|\Delta v\|_{L^{2}(\Omega)} \leqslant \Psi_{1}(t)
$$

where $\Psi_{1}$ is a increasing function of the time $t$ having the properties $\lim _{t \searrow 0} \Psi_{1}(t)=0, \lim _{t \nearrow T} \Psi_{1}(t)<\infty$ for all $T$ finite.

Henceforth $\Psi_{i}, i=2,3, \ldots$ will stand for a generic function of $t$ having the same properties as the function $\Psi_{1}$.

Lemma 4.11 Let $\Omega \subset \mathbb{R}^{2}$ be a bounded domain. If the hypothesis $(\mathcal{H})$ is satisfied, $v_{0} \in H^{1}(\Omega) \cap L^{\infty}(\Omega)$ and $w_{0} \in W^{1,4}(\Omega)$, then we have

$$
\|\nabla v\|_{L^{1}\left(0, t ; L^{p}(\Omega)\right)} \leqslant \Psi_{2}(t)
$$

for all $0<t<T$ and $2 \leqslant p<\infty$.

Proof. Taking into account the Gagliardo-Nirenberg inequality and the Cauchy inequality we obtain the following estimate

$$
\int_{0}^{t}\|\nabla v\|_{L^{2^{j}}(\Omega)} \leqslant \int_{0}^{t}\|\Delta v\|_{L^{2}(\Omega)}+\frac{C_{25}^{2}}{4} \int_{0}^{t}\|\nabla v\|_{L^{2^{j-1}}(\Omega)}
$$

for $j=2,3, \ldots$. The last inequality implies

$$
\int_{0}^{t}\|\nabla v\|_{L^{2^{j}(\Omega)}} \leqslant \frac{1-\left(\frac{C_{25}^{2}}{4}\right)^{j-1}}{1-\frac{C_{25}^{2}}{4}} \int_{0}^{t}\|\Delta v\|_{L^{2}(\Omega)}+\left(\frac{C_{25}^{2}}{4}\right)^{j-1} \int_{0}^{t}\|\nabla v\|_{L^{2}(\Omega)} .
$$

From the last inequality and using (4.43) and (4.49) we obtain (4.62) for $p=2^{j}, j=2,3, \ldots$. We conclude the lemma from (4.43) and taking into account the embeddings of $L^{p}(\Omega)$ spaces. 
Lemma 4.12 Let $\Omega \subset \mathbb{R}^{2}$ be a bounded domain. If the hypothesis $(\mathcal{H})$ is satisfied, $v_{0} \in H^{1}(\Omega) \cap L^{\infty}(\Omega)$ and, $w_{0} \in W^{1, p}(\Omega)$, then we have

$$
\|\nabla w\|_{L^{p}(\Omega)} \leqslant \Psi_{3}(t)
$$

for all $0<t<T$ and $2<p<\infty$.

Proof. We deduce from the equation (4.17)

$$
\nabla w_{t}=-e^{w} w^{\beta} v \nabla w-\beta e^{w} w^{\beta-1} v \nabla w-e^{w} w^{\beta} \nabla v .
$$

Multiplying this last relation by $\nabla w|\nabla w|^{p-2}$ and after that integrating in $\Omega \times(0, t)$, we have

$$
\|\nabla w\|_{L^{p}(\Omega)} \leqslant\left\|\nabla w_{0}\right\|_{L^{p}(\Omega)}+e^{\left\|w_{0}\right\|_{L^{\infty}(\Omega)}}\left\|w_{0}\right\|_{L^{\infty}(\Omega)}^{\beta}\|\nabla v\|_{L^{1}\left(0, t ; L^{p}(\Omega)\right)} .
$$

From the last inequality and Lemma 4.11 the statement follows.

We consider the equation (4.16) together with (4.18) and (4.19) like a linear problem in the variable $v$ in the general form (2.7)-(2.9), considering

$$
\begin{aligned}
b_{i}(x, t) & =\frac{\partial w}{\partial x_{i}}(x, t), \quad i=1,2 \\
b(x, t) & =-w_{t}+\delta\left(1+w_{t} w^{-\beta}\right) \\
\widetilde{F}(x, t) & =\widetilde{G}(x, t)=0 .
\end{aligned}
$$

Taking $\left(v_{0}, w_{0}\right) \in W^{2-2 / p, p}(\Omega) \times W^{1, \max \{p, 4\}}(\Omega), p \geqslant 2$ we observe, taking into account also the above estimates, that the hypotheses of (21, Theorem 9.1, cap. IV]) are fulfilled (see also [24, Theorem II.3]). This implies that for $p \geqslant 2$ we have $v \in W_{p}^{2,1}\left(\Omega_{T}\right)$. Moreover, taking into account the embedding results in Hölder spaces we obtain $v \in C^{2-4 / p, 1-2 / p}\left(\bar{\Omega}_{T}\right)$ and

$$
|v|_{\Omega_{T}}^{(2-4 / p)} \leqslant \Psi_{3}(t)
$$

for all $0<t<T$ and $2<p<\infty$.

Lemma 4.13 Let $\Omega \subset \mathbb{R}^{2}$ be a bounded domain. If the hypothesis $(\mathcal{H})$ is satisfied, $v_{0} \in H^{1}(\Omega) \cap L^{\infty}(\Omega)$ and $w_{0} \in W^{2, p}(\Omega), p>2$, then $w \in C^{2-4 / p, 1-2 / p}\left(\bar{\Omega}_{T}\right)$ and

$$
|w|_{\Omega_{T}}^{(2-4 / p)} \leqslant \Psi_{4}(t)
$$

for all $0<t<T$ and $2<p<\infty$.

Proof. We deduce from the equation (4.17)

$$
\Delta w_{t}=-e^{w}\left\{w^{\beta-1}\left[w \Delta v+(w+\beta)(2 \nabla v \cdot \nabla w+v \Delta w)+v(w+2 \beta)|\nabla w|^{2}\right]-v w^{\beta-2} \beta(\beta-1)|\nabla w|^{2}\right\} .
$$

We multiply the last relation by $(\Delta w)^{p-1}$ with $p=2^{j}, j=1,2, \ldots$ Integrating after that in $\Omega$ and applying Young's inequality we obtain

$$
\|\Delta w\|_{L^{p}(\Omega)}^{p} \leqslant e^{4(p-1) \varepsilon^{(p /(p-1)) t}}\left[\left\|\Delta w_{0}\right\|_{L^{p}(\Omega)}^{p}+\int_{0}^{t} M(s) d s\right]
$$


where

$$
\begin{aligned}
& \int_{0}^{t} M(s) d s=\varepsilon^{-p} e^{p\left\|w_{0}\right\|_{L^{\infty}(\Omega)}}\left[\left\|w_{0}\right\|_{L^{\infty}(\Omega)}^{p \beta}\left(\|\Delta v\|_{L^{1}\left(0, t ; L^{p}(\Omega)\right)}^{p}+2 \int_{0}^{t}\|\nabla v \cdot \nabla w\|_{L^{p}(\Omega)}^{p}\right)+\right. \\
& +2 \beta\left\|w_{0}\right\|_{L^{\infty}(\Omega)}^{p(\beta-1)}\left(\int_{0}^{t}\|\nabla v \cdot \nabla w\|_{L^{p}(\Omega)}^{p}+\|v\|_{L^{\infty}\left(0, t ; L^{\infty}(\Omega)\right)}^{p} \int_{0}^{t}\|\nabla w\|_{L^{2 p}(\Omega)}^{2 p}\right)+ \\
& \left.+\|v\|_{L^{\infty}\left(0, t ; L^{\infty}(\Omega)\right)}^{p}\left\|w_{0}\right\|_{L^{\infty}(\Omega)}^{p(\beta-2)}\left(\left\|w_{0}\right\|_{L^{\infty}(\Omega)}^{2}+\beta|\beta-1|\right)^{p} \int_{0}^{t}\|\nabla w\|_{L^{2 p}(\Omega)}^{2 p}\right]
\end{aligned}
$$

Next observe that Lemma 4.11, Lemma 4.12 and (4.63) allow us to estimate the integral on the right-hand side of (4.65) and to obtain

$$
\|\Delta w\|_{L^{p}(\Omega)}^{p} \leqslant e^{4(p-1) \varepsilon^{(p /(p-1)) t}}\left[\left\|\Delta w_{0}\right\|_{L^{p}(\Omega)}^{p}+\Psi_{4}(t)\right] .
$$

In a similar way, we obtain $D_{x}^{s} w \in L^{p}\left(\Omega_{T}\right),|s|=2$. Taking into account Lemma 4.12 and the embedding results in Hölder space we conclude the proof.

Lemma 4.14 Let $\Omega \subset \mathbb{R}^{2}$ be a bounded domain. If the hypothesis $(\mathcal{H})$ is satisfied and $\left(u_{0}, w_{0}\right) \in$ $\left(W^{2, p}(\Omega)\right)^{2}, p>2$, then $u \in C^{2-4 / p, 1-2 / p}\left(\bar{\Omega}_{T}\right)$ and

$$
|u|_{\Omega_{T}}^{(2-4 / q)} \leqslant \Psi_{5}(t)
$$

for all $T$ finite, $0<t<T$.

Proof. The conclusion of the Lemma follows from (4.63), (4.64) and Lemma 2.3.

To achieve the proof of Theorem 4.1 we use the next Lemma whose proof is similar to the proof of Lemmas IV.2 and IV.3 in [24] (see also Lemma 2 in [25]).

Lemma 4.15 (i) Suppose that $\|u\|_{\Omega_{T}}^{(m)} \leqslant \Psi(t), m>1, m$ not integer, for all $0 \leqslant t<T$. Then we have

$$
\left|w^{\beta} \Delta U\right|_{\Omega_{t}}^{(\alpha)}+\left|w^{\beta} \nabla U\right|_{\Omega_{t}}^{(\alpha)}+\left.\left.\left|w^{-1}\right| \nabla w\right|^{2}\right|_{\Omega_{t}} ^{(\alpha)} \leqslant \Psi(t)
$$

for all $0 \leqslant t<T$, where $\alpha=\min \{l+2, m-1\}$.

(ii) Let $\alpha>0$ not integer. If

$$
\left|w^{\beta} \Delta U\right|_{\Omega_{t}}^{(\alpha)}+\left|w^{\beta} \nabla U\right|_{\Omega_{t}}^{(\alpha)}+\left.\left.\left|w^{-1}\right| \nabla w\right|^{2}\right|_{\Omega_{t}} ^{(\alpha)} \leqslant \Psi(t)
$$

for all $0 \leqslant t<\tau$, then

$$
|u|_{\Omega_{t}}^{(\eta+2)} \leqslant \Psi(t)
$$

where $\eta=\min \{\alpha, l, m\}$.

In such a way the regularity of the solution $u$ is successively ameliorated until reaching the desired bound of $|u|_{\Omega_{t}}^{(l+2)}$. 


\section{$5 \quad$ Asymptotic behavior of global solutions}

\section{$5.1 \quad$ Steady states}

In this Section we are going to study the asymptotic behavior of the smooth solution of the problem (3.1)-(3.5). We shall begin by analyzing the steady states of the system (3.1)- (3.2) with homogeneous Neumann boundary condition (3.3). So, we consider the following stationary problem:

$$
\begin{array}{ll}
0=\Delta u-\nabla \cdot(u \nabla w)+\delta u(1-u) & x \in \Omega \\
0=w^{\beta} u & x \in \Omega \\
\frac{\partial u}{\partial \eta}-u \frac{\partial w}{\partial \eta}=0 & x \in \partial \Omega .
\end{array}
$$

Lemma 5.1 Let $\Omega \subset \mathbb{R}^{N}, N \geqslant 1$ be a domain. Let $u, w \in C^{1}(\Omega)$ be two functions satisfying uw $=0$, for all $x \in \Omega$. Then we have $\nabla u \cdot \nabla w=0$ for all $x \in \Omega$.

Proof. We consider the closed sets

$$
F=u^{-1}(0), \quad G=w^{-1}(0) .
$$

The fact that $u w=0$ implies that $F \cup G=\Omega$. As $F$ and $G$ are closed it is straightforward to show that

$$
\overline{\operatorname{int} F} \cup \overline{\operatorname{int} G}=\Omega \text {. }
$$

As the functions $u$ and $w$ belong to $C^{1}(\Omega)$, the sets $(\nabla u)^{-1}(0),(\nabla w)^{-1}(0)$ are closed. Moreover, $\operatorname{int} F \subset(\nabla u)^{-1}(0)$, int $G \subset(\nabla w)^{-1}(0)$, which imply, taking into account (5.4)

$$
\Omega \subset(\nabla u)^{-1}(0) \cup(\nabla w)^{-1}(0)
$$

and the proof is complete.

Proposition 5.2 Let $\Omega \subset \mathbb{R}^{N}, N \geqslant 1$ be an open set. If $(u, w) \in\left(C^{2}(\Omega) \cap C^{1}(\bar{\Omega})\right)^{2}$ is a classical solution to (5.1)-(5.3) then

$$
(u, w)=(0, \widetilde{w}) \quad \text { or } \quad(u, w)=(k, 0)
$$

where $\widetilde{w} \in C^{2}(\Omega) \cap C^{1}(\bar{\Omega})$ and $k$ is a constant if $\delta=0$ and $k=1$ if $\delta>0$.

Proof. If $\delta=0$, we multiply (5.1) by $u$ and integrate over $\Omega$. We obtain

$$
0=-\int_{\Omega}|\nabla u|^{2}+\int_{\Omega} u \nabla w \cdot \nabla u .
$$

The last equality and Lemma 5.1 imply that $u$ is a constant. Taking also into account (5.2)-(5.3), the conclusion of the theorem follows.

We now turn to the case $\delta>0$. Multiplying (5.1) by $u-1$ and integrating over $\Omega$ we obtain

$$
0=-\int_{\Omega}|\nabla u|^{2}+\int_{\Omega} u \nabla w \cdot \nabla u-\int_{\Omega} \delta u(u-1)^{2} .
$$

From Lemma 5.1 we have

$$
\int_{\Omega}|\nabla u|^{2}=-\int_{\Omega} \delta u(u-1)^{2}<0 .
$$

We conclude the proof using the same arguments as above.

In the remaining of this paper we shall place ourselves in the hypotheses of Theorem 4.1. Then the system (3.1)-(3.5) has a global in time classical Hölder continuous solution. We emphasize that the hypothesis $(\mathcal{H})$ is also fulfilled. 
Lemma 5.3 If there exists a positive constant $\gamma>0$ such that $u_{0}(x) \geqslant \gamma$ for all $x \in \Omega$, then every global solution $u$ of (3.1)-(3.5) satisfies $u(x, t) \geqslant \min \{1, \gamma\} e^{-\left\|w_{0}\right\|_{L} \infty(\Omega)}$ for all $x \in \Omega, t>0$.

Proof. Let $\alpha$ be a positive constant to be chosen later. By multiplying the equation (4.16) by $e^{w}(v-$ $\alpha)_{-}=e^{w} \max \{\alpha-v, 0\}$ and integrating over $\Omega$ we get

$$
\begin{aligned}
\frac{1}{2} \frac{d}{d t} \int_{\Omega} e^{w}(v-\alpha)_{-}^{2} & =-\int_{\Omega} e^{w}\left|\nabla(v-\alpha)_{-}\right|^{2}-\int_{\Omega} e^{2 w} v^{2} w^{\beta}(v-\alpha)_{-}- \\
& -\frac{1}{2} \int_{\Omega} e^{2 w} v w^{\beta}(v-\alpha)_{-}^{2}-\delta \int_{\Omega} e^{w} v\left(1-v e^{w}\right)(v-\alpha)_{-} .
\end{aligned}
$$

If $\delta=0$, let us observe that the right-hand side of (5.5) is nonpositive. If $\delta>0$, we choose $\alpha$ small enough

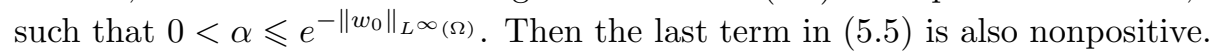

From the above considerations, we get

$$
\int_{\Omega} e^{w}(v-\alpha)_{-}^{2} \leqslant \int_{\Omega} e^{w_{0}}\left(v_{0}-\alpha\right)_{-}^{2} .
$$

We consider first the case when $\gamma<1$. We choose $\alpha=\gamma e^{-\left\|w_{0}\right\|_{L \infty}(\Omega)}<e^{-\left\|w_{0}\right\|_{L \infty}(\Omega)}$. Because $u_{0} \geqslant \gamma>0$, we obtain $v_{0}=u_{0} e^{-w_{0}} \geqslant \gamma e^{-\left\|w_{0}\right\|_{L}(\Omega)}=\alpha$. From (5.6) we obtain that $u \geqslant \gamma e^{-\left\|w_{0}\right\|_{L^{\infty}(\Omega)}}$.

Now let $\gamma \geqslant 1$. We choose $\alpha=e^{-\left\|w_{0}\right\|_{L}(\Omega)}$. Because $u_{0} \geqslant \gamma>1$, we obtain $v_{0}=u_{0} e^{-w_{0}} \geqslant e^{-\left\|w_{0}\right\|_{L^{\infty}(\Omega)}}=$ $\alpha$. From (5.6) we obtain that $u \geqslant e^{-\left\|w_{0}\right\|_{L^{\infty}(\Omega)}}$.

\subsection{Exponential convergence}

In this subsection we consider $\beta=1$.

Lemma 5.4 If there exists a positive constant $\gamma>0$ such that $u_{0}(x) \geqslant \gamma$ for all $x \in \Omega$, then

$$
\int_{\Omega}|\nabla w|^{2} \leqslant\left(C_{27}+C_{28} t\right) e^{-2 \lambda t}
$$

where $C_{27}, C_{28}$ are positive constants independent on $t$ and $\lambda=\min \{1, \gamma\} e^{-\left\|w_{0}\right\|_{L}(\Omega)}>0$.

Proof. From (3.2) we obtain

$$
|\nabla w|^{2} \leqslant 2 e^{-2 \int_{0}^{t} u}\left|\nabla w_{0}\right|^{2}+2 t e^{-2 \int_{0}^{t} u}\left|w_{0}\right|^{2} \int_{0}^{t}|\nabla u|^{2} .
$$

Taking into account Lemma 5.3 we know that $u(x, t) \geqslant \lambda>0$ for all $x \in \Omega, t>0$. We have from the previous inequality

$$
\int_{\Omega}|\nabla w|^{2} \leqslant 2 e^{-2 \lambda t} \int_{\Omega}\left|\nabla w_{0}\right|^{2}+2 t e^{-2 \lambda t}\left\|w_{0}\right\|_{L^{\infty}(\Omega)}^{2} \int_{0}^{t} \int_{\Omega}|\nabla u|^{2} .
$$

Taking into account the hypothesis $(\mathcal{H})$, the estimates (4.7), (4.12) and because every term of the functional $D(u, w)$ given by (4.6) is positive, we obtain that the last term in (5.8) is bounded. More precisely

$$
\int_{0}^{t} \int_{\Omega}|\nabla u|^{2} d x d s \leqslant\|u\|_{L^{\infty}\left(0, t ; L^{\infty}(\Omega)\right)} \int_{0}^{t} D(u, w) \leqslant C_{26}\|u\|_{L^{\infty}\left(0, t ; L^{\infty}(\Omega)\right)}
$$


where $C_{26}=F\left(u_{0}, w_{0}\right)+|\Omega|$. Finally, from (5.8) and (5.9) we obtain

$$
\int_{\Omega}|\nabla w|^{2} \leqslant 2 e^{-2 \lambda t}\left[\left\|\nabla w_{0}\right\|_{L^{2}(\Omega)}^{2}+C_{26} t\left\|w_{0}\right\|_{L^{\infty}(\Omega)}^{2}\|u\|_{L^{\infty}\left(0, t ; L^{\infty}(\Omega)\right)}\right]=\left(C_{27}+C_{28} t\right) e^{-2 \lambda t}
$$

and we conclude the proof.

Proposition 5.5 If there exists a positive constant $\gamma>0$ such that $u_{0}(x) \geqslant \gamma$ for all $x \in \Omega$, then

$$
\begin{array}{ll}
\|u(\cdot, t)-\bar{u}\|_{L^{2}(\Omega)} \leqslant\|u\|_{L^{\infty}\left(0, t ; L^{\infty}(\Omega)\right)}^{2}\left(C_{27}+\frac{C_{28}}{2} t\right) t e^{-C_{30} t}, & \text { if } \delta=0 \\
\|u(\cdot, t)-1\|_{L^{2}(\Omega)} \leqslant\left[\left\|u_{0}-1\right\|_{L^{2}(\Omega)}^{2}+\|u\|_{L^{\infty}\left(0, t ; L^{\infty}(\Omega)\right)}^{2}\left(C_{27}+\frac{C_{28}}{2} t\right) t\right] e^{-2 \lambda \min \{1, \delta\} t}, & \text { if } \delta>0 \\
\|w(\cdot, t)\|_{L^{\infty}(\Omega)} \leqslant\left\|w_{0}\right\|_{L^{\infty}(\Omega)} e^{-\lambda t} &
\end{array}
$$

where $\bar{u}=\frac{1}{|\Omega|} \int_{\Omega} u_{0}, \lambda=\min \{1, \gamma\} e^{-\left\|w_{0}\right\|_{L^{\infty}(\Omega)}}>0$ and $C_{27}, C_{28}, C_{30}$ are positive constants independent on $t$.

Proof. Let $\sigma$ be a positive constant to be chosen later. We multiply the equation (3.1) by $(u-\sigma)$ and integrate over $\Omega$

$$
\frac{d}{d t} \int_{\Omega}(u-\sigma)^{2} \leqslant-\int_{\Omega}|\nabla u|^{2}+\|u\|_{L^{\infty}\left(0, t ; L^{\infty}(\Omega)\right)}^{2} \int_{\Omega}|\nabla w|^{2}-2 \delta \int_{\Omega} u(u-1)(u-\sigma) .
$$

First we consider the case $\delta=0$ and $\sigma=\bar{u}=\frac{1}{|\Omega|} \int_{\Omega} u_{0}(x)$. Applying the Poincaré inequality in (5.10) we obtain

$$
\frac{d}{d t} \int_{\Omega}(u-\bar{u})^{2}+C_{29} \int_{\Omega}(u-\bar{u})^{2} \leqslant\|u\|_{L^{\infty}\left(0, t ; L^{\infty}(\Omega)\right)}^{2} \int_{\Omega}|\nabla w|^{2} .
$$

Applying the Gronwall inequality in the last estimate and taking into account (5.7) we have

$$
\int_{\Omega}(u-\bar{u})^{2} \leqslant\|u\|_{L^{\infty}\left(0, t ; L^{\infty}(\Omega)\right)}^{2}\left(C_{27}+\frac{C_{28}}{2} t\right) t e^{-C_{30} t}
$$

where $C_{30}=\min \left\{2 \lambda, C_{29}\right\}$.

Let now $\delta \neq 0$ and $\sigma=1$. Using Lemma 5.3 we obtain from (5.10)

$$
\frac{d}{d t} \int_{\Omega}(u-1)^{2}+2 \lambda \delta \int_{\Omega}(u-1)^{2} \leqslant\|u\|_{L^{\infty}\left(0, t ; L^{\infty}(\Omega)\right)}^{2} \int_{\Omega}|\nabla w|^{2} .
$$

Applying Gronwall's inequality and (5.7) it follows that

$$
\int_{\Omega}(u-1)^{2} \leqslant\left[\left\|u_{0}-1\right\|_{L^{2}(\Omega)}^{2}+\|u\|_{L^{\infty}\left(0, t ; L^{\infty}(\Omega)\right)}^{2}\left(C_{27}+\frac{C_{28}}{2} t\right) t\right] e^{-2 \lambda \min \{1, \delta\} t} .
$$

Finally, from (4.3) we obtain

$$
w=w_{0} e^{-\int_{0}^{t} u} \leqslant w_{0} e^{-\lambda t}
$$

and we conclude the proof. 


\subsection{Polynomial convergence}

In this subsection we consider $\beta>1$.

Lemma 5.6 If there exists a positive constant $\gamma>0$ such that $u_{0}(x) \geqslant \gamma$ for all $x \in \Omega$, then

$$
\int_{0}^{t}\left(\left\|w_{0}\right\|_{L^{\infty}(\Omega)}^{1-\beta}+\lambda(\beta-1) s\right)^{\frac{1}{\beta-1}} \int_{\Omega}|\nabla w|^{2} d x d s \leqslant C_{31}
$$

where $C_{31}$ is a positive constant independent on $t$ and $\lambda=\min \{1, \gamma\} e^{-\left\|w_{0}\right\|_{L \infty(\Omega)}}>0$.

Proof. Taking into account the hypothesis $(\mathcal{H})$ and the estimates (4.7) and (4.12) we have

$$
\int_{0}^{t} \int_{\Omega} u w^{-1}|\nabla w|^{2} d x d s \leqslant \int_{0}^{t} D(u, w) \leqslant C_{26}
$$

From Lemma 5.3 we know that $u(x, t) \geqslant \lambda>0$ for all $x \in \Omega, t>0$. Taking into account (4.3) we obtain

$$
u w^{-1} \geqslant \lambda\left(w_{0}^{1-\beta}+\lambda(\beta-1) t\right)^{\frac{1}{\beta-1}}
$$

The inequalities (5.14) and (5.15) imply

$$
\int_{0}^{t}\left(\left\|w_{0}\right\|_{L^{\infty}(\Omega)}^{1-\beta}+\lambda(\beta-1) s\right)^{\frac{1}{\beta-1}} \int_{\Omega}|\nabla w|^{2} d x d s \leqslant \lambda^{-1} \int_{0}^{t} \int_{\Omega} u w^{-1}|\nabla w|^{2} d x d s \leqslant \lambda^{-1} C_{26}
$$

and we conclude the proof with $C_{31}=\lambda^{-1} C_{26}$.

Proposition 5.7 If there exists a positive constant $\gamma>0$ such that $u_{0}(x) \geqslant \gamma$ for all $x \in \Omega$, then

$$
\begin{array}{ll}
\|u(\cdot, t)-\bar{u}\|_{L^{2}(\Omega)} \leqslant C_{32}\left(\left\|w_{0}\right\|_{L^{\infty}(\Omega)}^{1-\beta}+\lambda(\beta-1) t\right)^{-\frac{1}{\beta-1}}, & \text { if } \delta=0 \\
\|u(\cdot, t)-1\|_{L^{2}(\Omega)} \leqslant C_{33}\left(\left\|w_{0}\right\|_{L^{\infty}(\Omega)}^{1-\beta}+\lambda(\beta-1) t\right)^{-\frac{1}{\beta-1}}, & \text { if } \delta>0 \\
\|w(\cdot, t)\|_{L^{\infty}(\Omega)} \leqslant\left[\left\|w_{0}\right\|_{L^{\infty}(\Omega)}^{1-\beta}+\lambda(\beta-1) t\right]^{-\frac{1}{\beta-1}} &
\end{array}
$$

where $\bar{u}=\frac{1}{|\Omega|} \int_{\Omega} u_{0}, \lambda=\min \{1, \gamma\} e^{-\left\|w_{0}\right\|_{L^{\infty}(\Omega)}}>0$ and $C_{32}, C_{33}$ are positive constants independent on $t$.

Proof. First we consider the case $\delta=0$.

Let us observe that for $t>t_{01}=\max \left\{0, \frac{1}{\lambda(\beta-1)}\left(\frac{\lambda}{C_{29}}-\left\|w_{0}\right\|_{L^{\infty}(\Omega)}^{1-\beta}\right)\right\}$ we have

$$
\begin{aligned}
& \frac{d}{d t}\left[\left(\left\|w_{0}\right\|_{L^{\infty}(\Omega)}^{1-\beta}+\lambda(\beta-1) t\right)^{\frac{1}{\beta-1}} \int_{\Omega}(u-\bar{u})^{2} d x\right]= \\
& =\left(\left\|w_{0}\right\|_{L^{\infty}(\Omega)}^{1-\beta}+\lambda(\beta-1) t\right)^{\frac{1}{\beta-1}}\left[\frac{d}{d t} \int_{\Omega}(u-\bar{u})^{2} d x+\lambda\left(\left\|w_{0}\right\|_{L^{\infty}(\Omega)}^{1-\beta}+\lambda(\beta-1) t\right)^{-1} \int_{\Omega}(u-\bar{u})^{2} d x\right] \leqslant \\
& \leqslant\left(\left\|w_{0}\right\|_{L^{\infty}(\Omega)}^{1-\beta}+\lambda(\beta-1) t\right)^{\frac{1}{\beta-1}}\left[\frac{d}{d t} \int_{\Omega}(u-\bar{u})^{2}+C_{29} \int_{\Omega}(u-\bar{u})^{2}\right] .
\end{aligned}
$$


We multiply (5.11) by $\left(\left\|w_{0}\right\|_{L^{\infty}(\Omega)}^{1-\beta}+\lambda(\beta-1) t\right)^{\frac{1}{\beta-1}}$ and then we integrate between $t_{01}$ and an arbitrary $t>t_{01}$. Taking into account (5.13) and (5.16) we have

$$
\int_{\Omega}(u-\bar{u})^{2} d x \leqslant C_{33}\left(\left\|w_{0}\right\|_{L^{\infty}(\Omega)}^{1-\beta}+\lambda(\beta-1) t\right)^{-\frac{1}{\beta-1}}
$$

where $C_{32}=\frac{1}{2 \varepsilon} C_{31}\|u\|_{L^{\infty}\left(0, t ; L^{\infty}(\Omega)\right)}^{2}+\left(\left\|w_{0}\right\|_{L^{\infty}(\Omega)}^{1-\beta}+\lambda(\beta-1) t_{01}\right)^{\frac{1}{\beta-1}} \int_{\Omega}\left(u\left(x, t_{01}\right)-\bar{u}\right)^{2} d x$.

If $\delta \neq 0$ we have for $t>t_{02}=\max \left\{0, \frac{1}{\lambda(\beta-1)}\left(\frac{1}{2 \delta}-\left\|w_{0}\right\|_{L^{\infty}(\Omega)}^{1-\beta}\right)\right\}$

$$
\begin{aligned}
& \frac{d}{d t}\left[\left(\left\|w_{0}\right\|_{L^{\infty}(\Omega)}^{1-\beta}+\lambda(\beta-1) s\right)^{\frac{1}{\beta-1}} \int_{\Omega}(u-1)^{2} d x\right] \leqslant \\
& \leqslant\left(\left\|w_{0}\right\|_{L^{\infty}(\Omega)}^{1-\beta}+\lambda(\beta-1) s\right)^{\frac{1}{\beta-1}}\left[\frac{d}{d t} \int_{\Omega}(u-1)^{2}+2 \lambda \delta \int_{\Omega}(u-1)^{2}\right] .
\end{aligned}
$$

Multiplying (5.12) by $\left(\left\|w_{0}\right\|_{L^{\infty}(\Omega)}^{1-\beta}+\lambda(\beta-1) t\right)^{\frac{1}{\beta-1}}$, integrating between $t_{02}$ and an arbitrary $t>t_{02}$ and taking into account the last inequality and (5.13), we obtain

$$
\int_{\Omega}(u-1)^{2} d x \leqslant C_{33}\left(\left\|w_{0}\right\|_{L^{\infty}(\Omega)}^{1-\beta}+\lambda(\beta-1) t\right)^{-\frac{1}{\beta-1}}
$$

where $C_{33}=\frac{1}{2 \varepsilon} C_{31}\|u\|_{L^{\infty}\left(0, t ; L^{\infty}(\Omega)\right)}^{2}+\left(\left\|w_{0}\right\|_{L^{\infty}(\Omega)}^{1-\beta}+\lambda(\beta-1) t_{02}\right)^{\frac{1}{\beta-1}} \int_{\Omega}\left(u\left(x, t_{02}\right)-1\right)^{2} d x$.

From (4.3) we obtain

$$
w=\left[w_{0}^{1-\beta}+(\beta-1) \int_{0}^{t} u\right]^{\frac{1}{1-\beta}} \leqslant\left[w_{0}^{1-\beta}+\lambda(\beta-1) t\right]^{\frac{1}{1-\beta}}
$$

and we conclude the proof.

\section{Acknowledgements}

This work was partially supported by the RTN "Modeling, Mathematical Methods and Computer Simulation of Tumour Growth and Therapy" (MRTN-CT-2004-503661). The first author was also partially supported by projects DGES (Spain) Grant MTM2007-61755 and CEEX Grant 05-D11-36/05.10.05.

\section{References}

[1] N. D. Alikakos, $L^{p}$ bounds of solutions of reaction-diffusion equations, Comm. Partial Differential Equations, 4 (1979), 827-868.

[2] A. R. A. Anderson, A hibrid mathematical model of solid tumour invasion: The importance of cell adhesion, IMA J. Math. Med. Biol., 22 (2005), 163-186.

[3] A. R. A. Anderson, M. Chaplain, Continuous and discrete mathematical models of tumour-induced angiogenesis, Bull. Math. Biol., 60 (1998), 857-900. 
[4] M. Chaplain, Mathematical modelling of tissue invasion, Cancer Modelling and Simulation, Chapter 10, ed. L. Preziosi, Chapman Hall/CRC, (2003), 269-297.

[5] L. Corrias, B. Perthame and H. Zaag, A chemotaxis model motivated by angiogenesis, C.R. Math. Acad. Sci. Paris, Ser. I, 336 (2003), 141-146.

[6] L. Corrias, B. Perthame and H. Zaag, Global solutions in some chemotaxis and angiogenesis systems in high space dimensions, Milan J. Math., 72 (2004), 1-28.

[7] L. Corrias, B. Perthame and H. Zaag, $L^{p}$ and $L^{\infty}$ a priori estimates for some chemotaxis models and aplications to the Cauchy problem, The mechanism of the spatio-temporal pattern arising in reaction diffusion system, Kyoto, (2004).

[8] W. Fang, K. Ito, On the time-dependent drift-diffusion model for semiconductors, J. Differential Equations, 117 (1995), 245-280.

[9] M. A. Fontelos, A. Friedman, B. Hu, Mathematical analysis of a model for the initiation of angiogenesis, SIAM J. Math. Anal., 33 (2002), 1330-1355.

[10] A. Friedman, J.I. Tello, Stability of solutions of chemotaxis equations in reinforced random walks, J. Math. Anal. Appl., 272 (2002), 138-163.

[11] H. Gajewski, K. Zacharias, Global behaviour of a reaction-diffusion system modelling chemotaxis, Math. Nachr., 195 (1998), 77-114.

[12] F. R. Guarguaglini, R. Natalini, Global existence of solutions to a nonlinear model of sulphation phenomena in calcium carbonate stones, Nonlinear Anal. Real World Appl., 6 (2005), 477-494.

[13] F. R. Guarguaglini, R. Natalini, Global existence and uniqueness of solutions for multidimensional weakly parabolic systems arising in chemistry and biology, Comm. Pure Appl. Anal., 6 (2007), 287-309.

[14] D. Horstmann, Lyapunov functions and $L^{p}$ estimates for a class of reaction-diffusion systems, Coll. Math., 87 (2001), 113-127.

[15] D. Horstmann, From 1970 until present: the Keller-Segel model in chemotaxis and its consequences I., Jahresber. Deutsch. Math.-Verein., 105 (2003), 103-165.

[16] D. Horstmann, From 1970 until present: the Keller-Segel model in chemotaxis and its consequences II., Jahresber. Deutsch. Math.-Verein., 106 (2004), 51-69.

[17] E. F. Keller, G. M. Odell, Necessary and sufficient conditions for chemotactic bands, Math. Biosci., 27 (1975), 309-317.

[18] E. F. Keller, G. M. Odell, Traveling bands of chemotactic bacteria revisited, J. Theoret. Biol., 56 (1976), 243-247.

[19] E.F. Keller and L.A. Segel, Initiation of slime mold agregation viewed as instability, J. Theoret. Biology, 26 (1970), 399-415.

[20] E. F. Keller, L. A. Segel, Traveling bands of chemotactic bacteria: a theoretical analysis, J. Theoret. Biol. 30 (1971), 235-248.

[21] O. A. Ladyženskaja, V. A. Solonnikov, N. N. Ural'ceva Linear and Quasi-linear Equations of Parabolic Type, Translation of Mathematical Monographs, vol. 23, American Mathematical Society, 1968.

[22] N. V. Mantzaris, S. Webb and H. G. Othmer, Mathematical modelling of tumour induced angiogenesis, J. Math. Biol., 49 (2004), 111-187. 
[23] A. J. Perumpanani, J. A. Sherratt, J. Norbury and H. M. Byrne, A two parameter family of travelling waves with a singular barrier arising from the modelling of matrix mediated malignant invasion. Phys. D., 126 (1999), 145-159.

[24] M. Rascle, Sur un équation intégro-différentielle non linéaire issue de la biologie, J. Diff. Eq. 32 (1979), 420-453.

[25] M. Rascle, On a system of non linear strongly coupled partial differential equations arising in Biology, Lectures Notes in Math. 846, Everitt and Sleeman eds., Springer-Verlag, New-York (1980), 290-298.

[26] G. Reyes, J. L. Vázquez, A weighted symmetrization for nonlinear elliptic and parabolic equations in inhomogeneous media, J. Eur. Math. Soc., 8 (2006), 531-554.

[27] J. I. Tello, Mathematical analysis and stability of a chemotaxis model with logistic term, Math. Methods Appl. Sci., 27 (2004), 1865-1880.

[28] C. Walker, Global well-posedness of a haptotaxis model including age and spatial structure, Diff. Int. Eq., 20 (2007), 1053-1074.

[29] C. Walker, G. F. Webb, Global existence of classical solutions for a haptotaxis model, SIAM J. Math. Anal., 38 (2007), 1694-1713. 